\title{
Least Square-Support Vector Machine Based Modeling for Electric Arc Furnaces
}

Leyla Vakilian

Bachelor of Computer Engineering, Shahid Beheshti University, 2001

\author{
A thesis \\ Presented to Ryerson University \\ in partial fulfillments of the degree of \\ Master of Science \\ In the program of \\ Computer Science
}

Toronto, Ontario, Canada, 2014

(C) Leyla Vakilian 2014 


\section{Author's Declaration}

I hereby declare that I am the sole author of this thesis. This is a true copy of the thesis, including any required final revisions, as accepted by my examiners.

I authorize Ryerson University to lend this thesis to other institutions or individuals for the purpose of scholarly research

I further authorize Ryerson University to reproduce this thesis by photocopying or by other means, in total or in part, at the request of other institutions or individuals for the purpose of scholarly research.

I understand that my thesis may be made electronically available to the public. 


\title{
Least Square-Support Vector Machine Based Modeling for
}

\section{Electric Arc Furnaces}

\author{
Leyla Vakilian \\ Master of Science \\ Department of Computer Science \\ Ryerson University, Toronto, Ontario, Canada, 2014
}

\begin{abstract}
The process of making steel from the scrap metal by means of electric arc furnaces (EAF) has been used extensively in the industry. Accurate modelling of EAFs is, therefore, desired to assess their operations and their impacts on the electrical network. A number of approaches have already been used to model the $v-i$ behavior of electric arc furnaces including mathematical methods and data-driven models. The objective of this thesis is to investigate the data-driven modelling methodologies, in particular, least square support vector machine (LS-SVM). The results obtained show that the proposed method with radial base function kernel provides the model to predict both arc current and arc voltage of EAFs.
\end{abstract}




\section{Acknowledgments}

I would like to express my deepest gratitude to my supervisor, Dr. Alireza Sadeghian, for his guidance, patience, insightful discussions, and providing me with the best atmosphere to conduct my research. One simply could not wish for a better supervisor.

I am very thankful to my thesis committee, Dr. Miri, Dr. Woungang, and Dr. Miranskyy for reading my thesis and their invaluable comments.

I cannot express enough thanks to my family especially my parents and my sister for their unconditional supports they provided me through my entire life. My parents and my sister have been my best friends all my life and I love them dearly and thank them for all their advices and supports. I also thank my brother-in-law for his great support.

Sincere thanks to all my friends specially Dr. Maryam Davoudpour, Mahsa Mostowfi, Graham Holker, Sweeney Luis, Olivia-Linda Enciu, Misagh Aghajani, Mike Papanicolau, and Shermineh Ghasemi for their kindness and moral support during my study. Thanks for the friendship and memories.

Last, but not least, I would like to thank my dearest friend, Ervis Sofroni, for helping me get through the difficult times. He is always there cheering me up and stood by me through the good times and bad.

Leyla Vakilian

Ryerson University

September 9, 2014 
Dedication

To my parents. 


\section{Table of Contents}

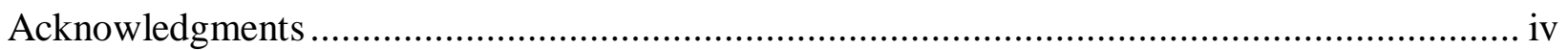

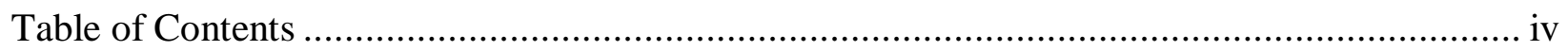

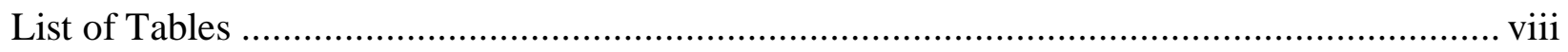

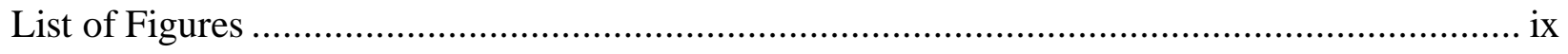

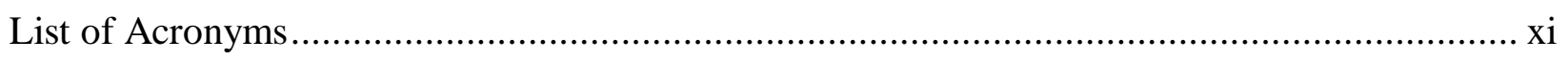

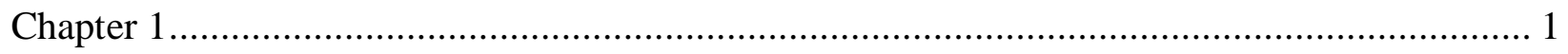

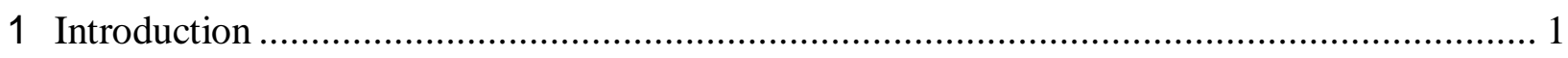

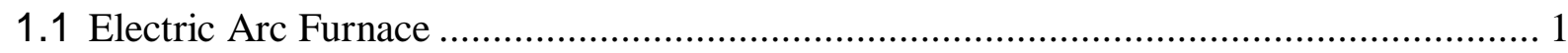

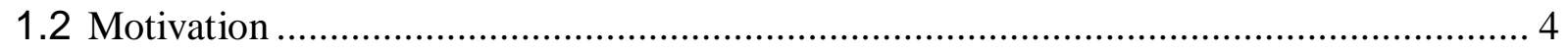

1.3 Least Square Support Vector Machine ……………............................................... 5

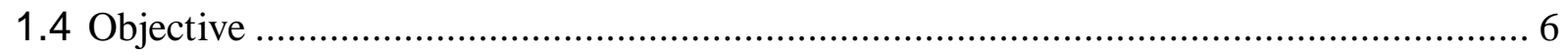

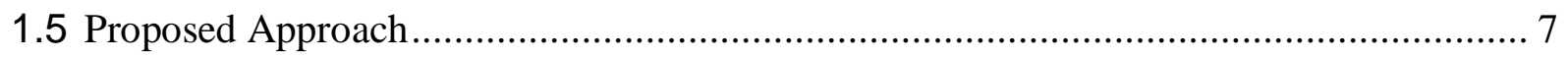

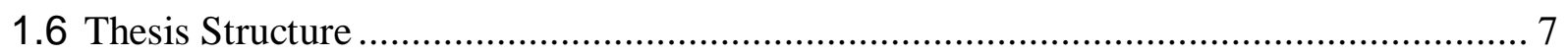

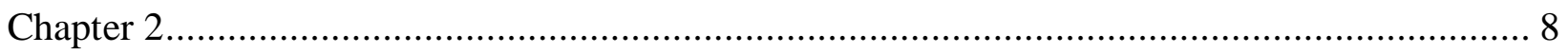

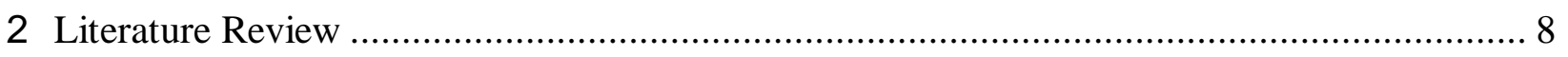

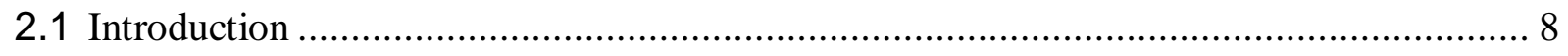

2.2 Structure and principal of EAF operation …………................................................... 8

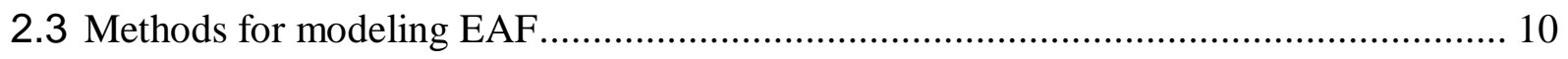

2.3.1 Mathematical Models ................................................................................ 10

2.3.2 Data-Driven Models (DDM) ................................................................... 19

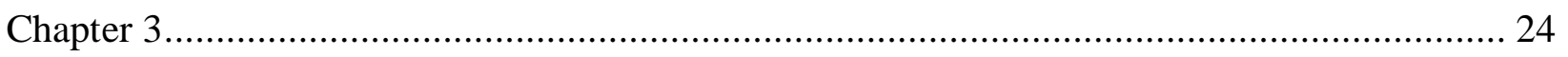

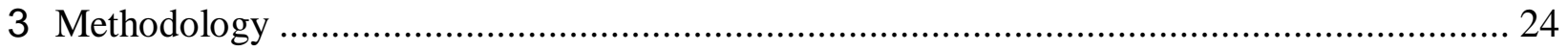

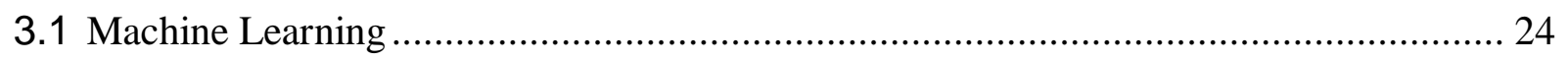

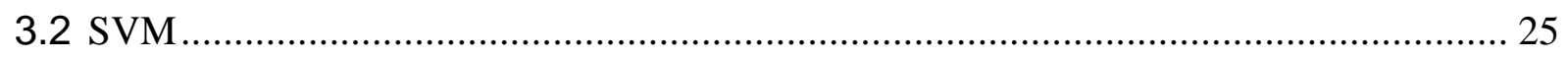




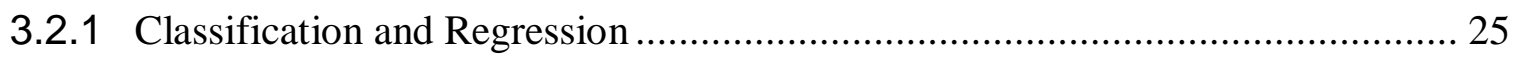

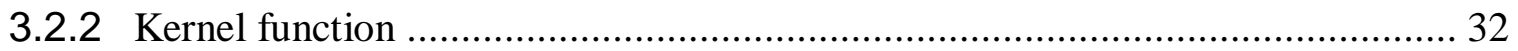

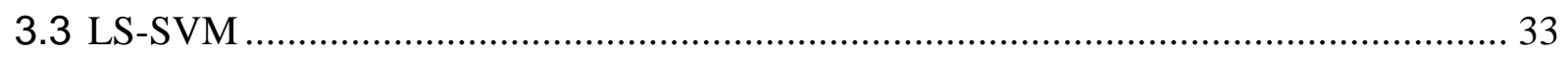

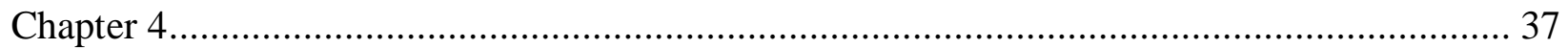

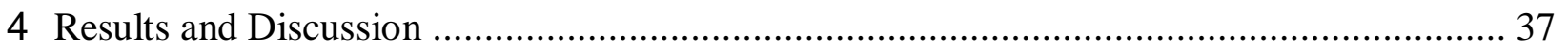

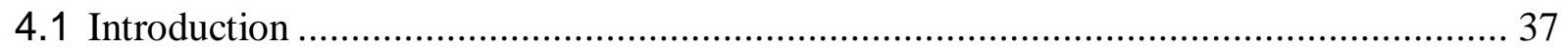

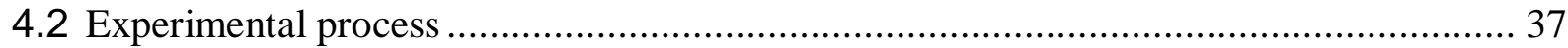

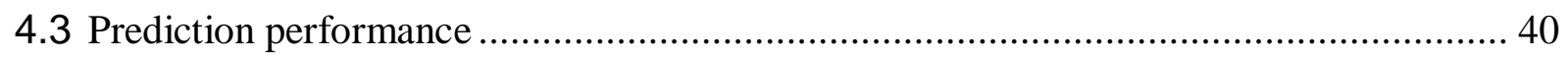

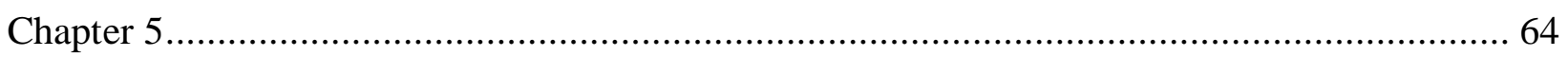

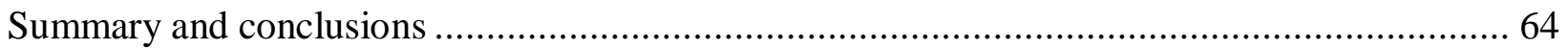

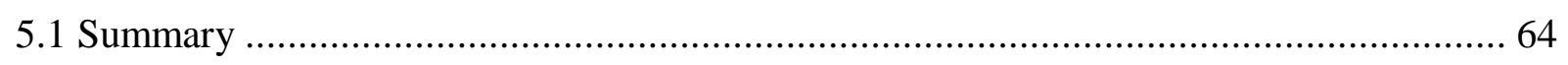

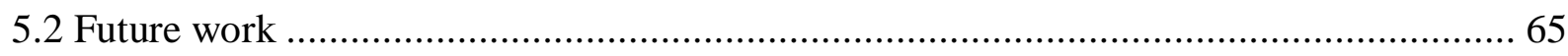

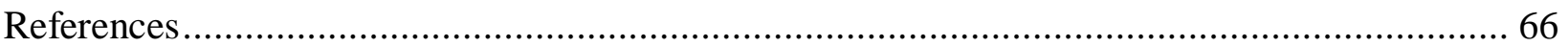




\section{List of Tables}

Table 4.1. Comparison between prediction the $v-i$ with different dimension of ..................... 48

Table 4.2. Comparison the results with different inputs............................................. 50

Table 4.3. Comparison of the slope of the best fit line and $95 \%$ confidence intervals .............. 58

Table 4.4. Comparison of the slope of best fit line and $95 \%$ confidence intervals between two best

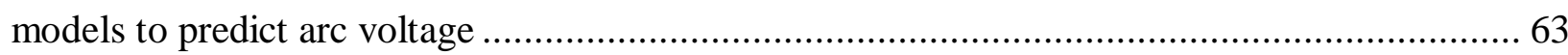




\section{List of Figures}

Fig. 1.1. Electrical Arc Furnace [1] ........................................................................ 2

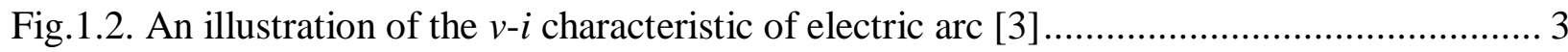

Fig. 1.3. Ideal Arc Voltage and Current Variation in Time [3] ........................................... 3

Fig. 3.1. Optimal Hyperplane of two data set .......................................................... 29

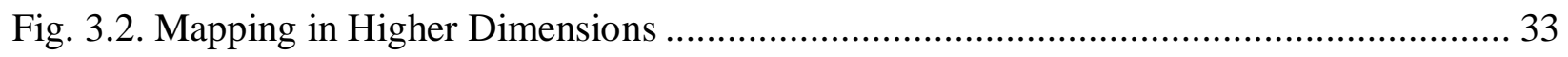

Fig. 4.1. Process of Building the LS-SVM Model ........................................................ 39

Fig. 4.4. LS-SVM input/output structure ............................................................ 41

Fig. 4.5. Actual data used as the training set .......................................................... 42

Fig. 4.6. Actual data used as the test set ........................................................................ 43

Fig. 4.7. Comparison of the predicted with the actual measurements for arc current and arc

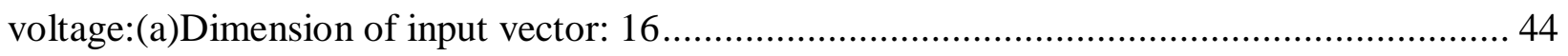

Fig. 4.8. Comparison of the predicted with the actual measurements for arc current and arc

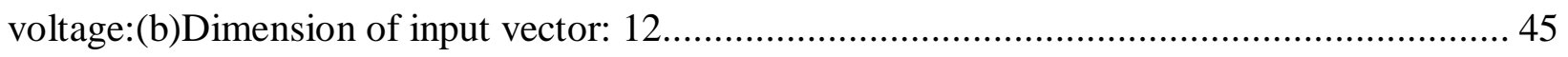

Fig. 4.9. Comparison of the predicted with the actual measurements for arc current and arc

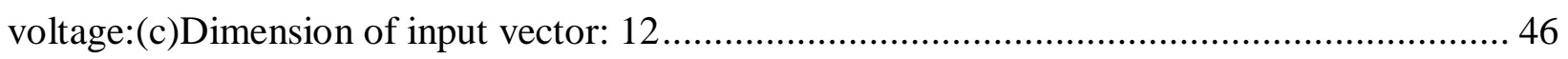

Fig. 4.10. Comparison of the predicted with the actual measurements for arc current and arc

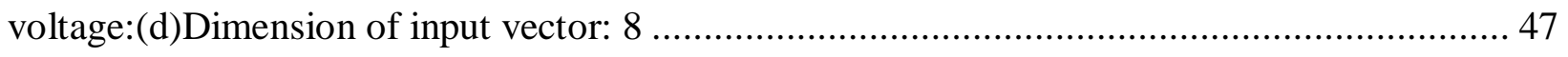

Fig. 4.11. New LS-SVM input/output structure...................................................... 49

Fig. 4.12. Comparison of the predicted vs actual measurements for arc current where the input is:(a) $\left[i_{t}, v_{t}\right]$, (b) $\left[i_{t-1}, i_{t} ; v_{t-1}, v_{t}\right]$ (c) $\left[i_{t-2}, i_{t-1}, i_{t} ; v_{t-2}, v_{t-1}, v_{t}\right]$, (d) $\left[i_{t-3}, i_{t-2}, i_{t-1}, i_{t} ; v_{t-3}, v_{t-2}, v_{t-1}, v_{t}\right] \ldots \ldots \ldots . . .51$

Fig. 4.13. Comparison of the predicted and actual values for arc voltage where input is........... 52

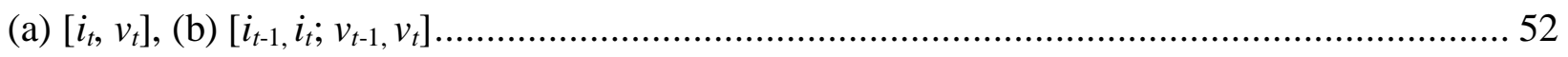


(c) $\left[i_{t-2}, i_{t-1}, i_{t} ; v_{t-2}, v_{t-1}, v_{t}\right]$, (d) $\left[i_{t-3}, i_{t-2}, i_{t-1}, i_{t} ; v_{t-3}, v_{t-2}, v_{t-1}, v_{t}\right]$

Fig. 4.14. Linear regression with the corresponding 95\% confidence interval between predicted and actual data for arc current using $\left[i_{t} ; v_{t}\right]$ as an input vector 54 Fig. 4.15. Linear regression with the corresponding 95\% confidence interval between predicted and actual data for arc current using $\left[i_{t-1}, i_{t} ; v_{t-1}, v_{t}\right]$ as an input vector...... 55

Fig. 4.16. Linear regression with the corresponding 95\% confidence interval between predicted and actual data for arc current using $\left[i_{t-2}, i_{t-1}, i_{t} ; v_{t-2}, v_{t-1}, v_{t}\right]$ as an input vector. 56 Fig. 4.17. Linear regression with the corresponding 95\% confidence interval between predicted and actual data for arc current using $\left[i_{t-3,}, i_{t-2}, i_{t-1}, i_{t} ; v_{t-3}, v_{t-2}, v_{t-1}, v_{t}\right]$ as an input vector.. 57 Fig. 4.18. Linear regression with the corresponding 95\% confidence interval between predicted and actual data for arc voltage using $\left[i_{t} ; v_{t}\right]$ as an input vector. 59

Fig. 4.19. Linear regression with the corresponding 95\% confidence interval between predicted and actual data for arc voltage using $\left[i_{t-1}, i_{t} ; v_{t-1}, v_{t}\right]$ as an input vector 60 Fig. 4.20. Linear regression with the corresponding 95\% confidence interval between predicted and actual data for arc voltage using $\left[i_{t-2}, i_{t-1}, i_{t} ; v_{t-2}, v_{t-1}, v_{t}\right]$ as an input vector 61 Fig. 4.21. Linear regression with the corresponding 95\% confidence interval between predicted and actual data for arc voltage using $\left[i_{t-3}, i_{t-2}, i_{t-1}, i_{t} ; v_{t-3}, v_{t-2}, v_{t-1}, v_{t}\right]$ as an input vector . 62 Fig. 5.1. LS-SVM input/output structure. 65 


\section{List of Acronyms}

\begin{tabular}{|c|c|}
\hline AI & Artificial Intelligence \\
\hline ANFIS & Adaptive Neuro-Fuzzy Inference System \\
\hline ANN & Artificial Neural Network \\
\hline CVS & Controlled Voltage Source \\
\hline CWT & Continuous Wavelet Transform \\
\hline DDM & Data Driven Model \\
\hline DRI & Direct Reduced Iron \\
\hline EAF & Electric Arc Furnace \\
\hline EMTP & Electro Magnetic Transients Program \\
\hline ERM & Empirical Risk Minimization \\
\hline FFT & Fast Fourier Transform \\
\hline LS-SVM & Least Square Support Vector Machine \\
\hline MAPD & Mean Absolute Percentage Deviation \\
\hline MAPE & Mean Absolute Percentage Error \\
\hline MAPFM & Maximum Apparent Power Fluctuation Method \\
\hline MLP & Multi-Layer Perceptron \\
\hline NDEI & Non-Dimensional Index Error \\
\hline PAH & Polycyclic Aromatic Hydrocarbons \\
\hline PSD & Power Spectral Density \\
\hline $\mathrm{RBF}$ & Radial Basis Function \\
\hline RBFN & Radial Basis Function Network \\
\hline
\end{tabular}




$\begin{array}{ll}\text { RME } & \text { Relative Mean Error } \\ \text { RMSE } & \text { Root Mean Squared Error } \\ \text { SRM } & \text { Structural Risk Minimization } \\ \text { SVM } & \text { Support Vector Machine } \\ \text { SVR } & \text { Support Vector Regression }\end{array}$




\section{Chapter 1}

\section{Introduction}

Different steel making technologies are employed by the industry to produce steel from iron and recycled scrap metals. A typical example includes basic oxygen steelmaking or the oxygen converter process in which molten pig iron or scrap metal from a blast furnace is converted to steel. The other widely used technology is electric arc furnace (EAF) that is mainly used in recycling to produce various grades of steel from scrap metal.

Due to the increased demand for production of steel by electric arc furnaces, accurate electric arc furnace models are needed to study the behavior and the impact of the EAFs on existing networks. This thesis describes a model for prediction the behavior of electric arc furnace (EAF) with least square support vector machine (LS-SVM).

\subsection{Electric Arc Furnace}

An electric arc furnace is a high-temperature furnace that makes steel by using high-voltage electric arcs. The EAF is the major component in scrap recycling process. In scrap metal recycling, steel is produced by melting scrap or direct reduced iron (DRI) by means of electric arcs in either AC or DC mode.

Fig. 1.1 shows different parts of the EAF. A typical EAF consists of three parts: the hearth, the shell, and the roof. Three carbon electrodes are located in the roof by a clamping mechanism. 
This mechanism makes each electrode lifts and lowers independently. The electrodes provide the current for the process.

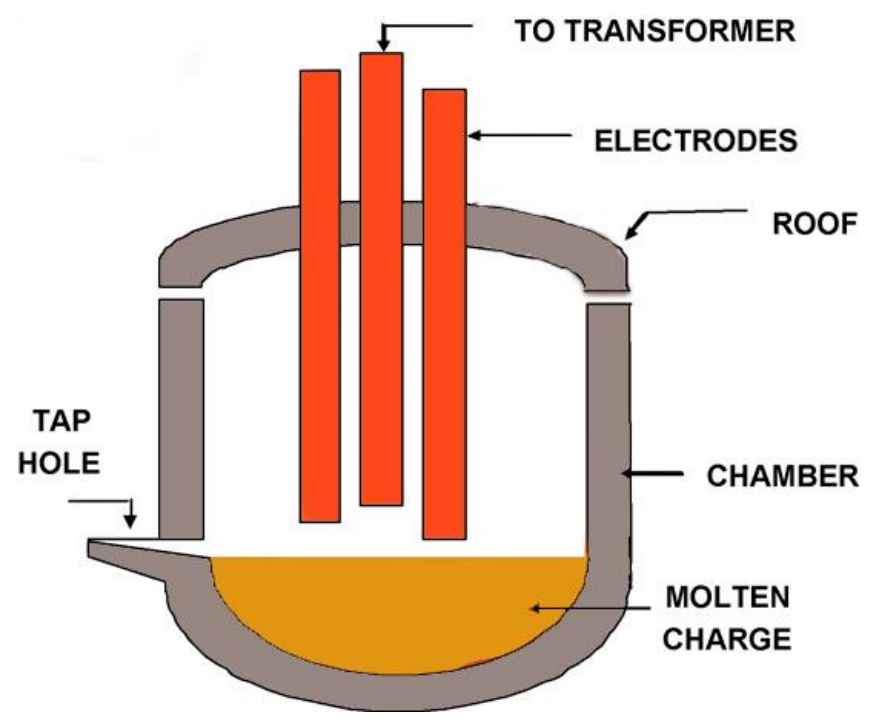

Fig. 1.1. Electrical Arc Furnace [1]

The graphite electrodes make arcs, which generate heat. The heat generated from arcs between graphite electrodes is the major source of energy to melt the steels. The voltage increases till the arc hits at the ignition voltage, $v_{i g}$, after striking the $v_{i g}$, the voltage descends to a constant value $v_{\text {arc }}$. Then the voltage rises to the extinguish voltage, $v_{e x}$. Increasing current is accompanied by an increasing arc voltage. At first the arc current, $i_{\text {arc }}$, is zero. After the arc ignites, the $i_{\text {arc }}$ has sinusoidal waveform. However when the arc extinguishes at $v_{e x}$, the $i_{\text {arc }}$ becomes zero [2]. The $v-i$ characteristic of the EAFs builds on the arc voltage and current waveforms (Fig. 1.2). 


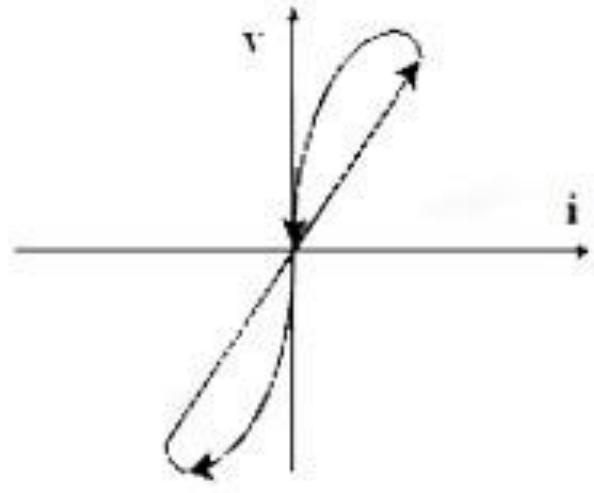

Fig.1.2. An illustration of the $v-i$ characteristic of electric arc [3]

In fact, the voltage can be illustrated as a function of the current and past history of the current. Fig. 1.3 demonstrates an ideal arc voltage and current variation in time.

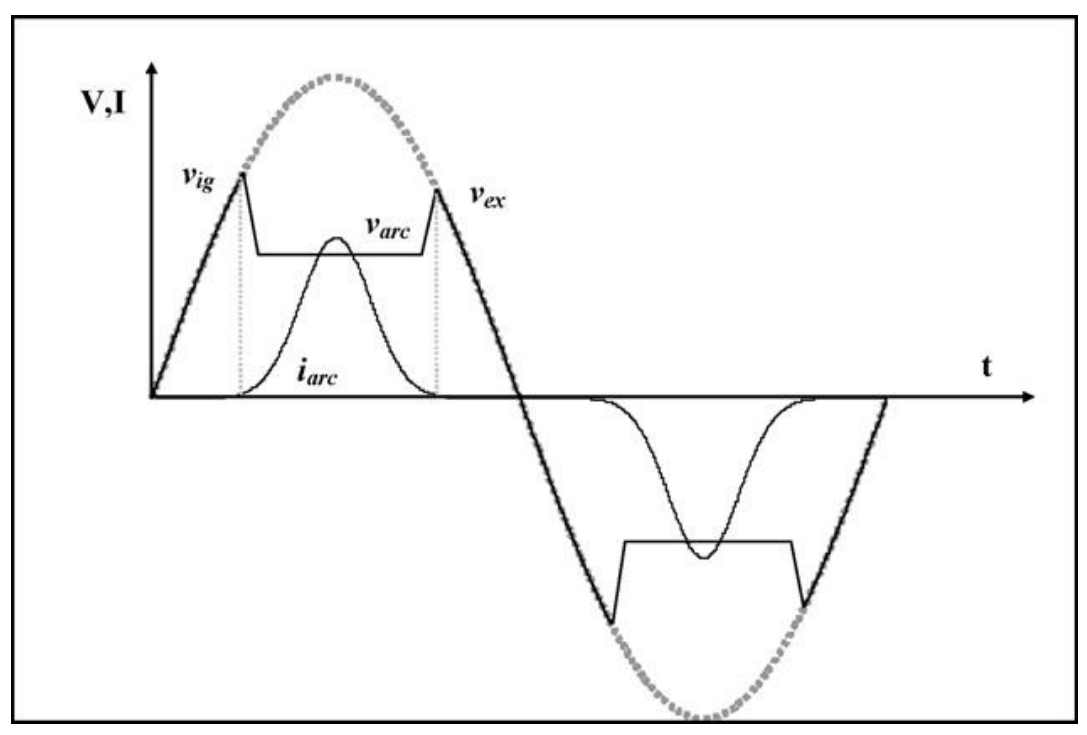

Fig. 1.3. Ideal Arc Voltage and Current Variation in Time [3] 


\subsection{Motivation}

Electric arc furnaces are widely used in the production of steel alloys and most importantly the stainless steel. Steel industries can reduce the environmental impacts such as carbon dioxide emissions, sulfur dioxide emissions, polycyclic aromatic hydrocarbons (PAH) emissions, waste going to landfill and also they can improve energy efficiency. Also Steel's recycling is the most recycled material in the world. In Canada, scrap metals significantly have value in economics because the metal can be kept its physical properties after recycling. Since The EAF uses $95 \%$ recycled scrap, it is necessary to have an accurate model for predicting the behaviour of the EAF. More accurate the system will be, more pollution minimizes and more natural environment protect we will have in future.

One of the important concerns in electric arc furnace is voltage flicker caused from variations of the large furnace loads. Another concern in electric arc furnaces is harmonics of frequency, which is caused by arc ignition delays and $v-i$ characteristics such as non-linearity, variation and non-consistency, highly, noisy environment.

The major objective of this thesis is to propose and investigate an approach to model the behaviour of the EAFs. Since the EAF is a non-linear and time varying system in reality, the data-driven methods, in particular the least square support vector machines are of main interest in this work. Support vector machines and least square support vector machines are to be used for regression, and modeling of the EAF, respectively. 


\subsection{Least Square Support Vector Machine}

Support vector machines (SVM) and least squares support vector machines (LS-SVM) are supervised learning techniques, which have successfully been used in non-linear classification and function estimation. SVM are based on statistical learning theory developed by Vapnik [4]. LS-SVMs, the modified versions of SVMs, were developed by Suykens and Vandewalle [5]. SVM and LS-SVM have been used for many learning tasks such as pattern recognition, regression analysis and object classification. The goal of the SVM and LS-SVM algorithms for classification is to build hyperplane that divides the data into two classes with the maximum margin, which is the maximum distance between the hyperplane and the closest examples.

Furthermore, the goal in SVM and LS-SVM regression is to evaluate an unknown function build on the number set of samples. To obtain this goal, the input of the non-linear system is mapped to a high-dimensional feature space to construct a linear model by using kernel functions. although both kernel based estimation methods build a linear model, the SVM builds a model by solving a quadratic programming problem in dual space [6] and LS-SVM builds the estimation function by solving a system of linear equations [7].

In recent years, there have been several applications in different aspects of SVM and LS-SVM. These aspects are based on theoretical understanding for real life events. The SVM is popular in solving the classification problem because it can be easily used and it is very flexible in class boundaries. The SVM can reduce the complexity and over-fitting of the model and also has good generalization performances. Since the SVM often has a good generalization performance, it has been used in different problems including text recognition, image detection, speech recognition, and emotion recognition bioinformatics and also it has been applied in many financial and time- 
prediction applications including time series prediction, travel-time prediction, bus arrival time prediction, credit rating analysing, and industrial process identifications.

In reality, industrial processes such as electric arc furnaces are time varying and nonlinear. So it is difficult to model EAFs behaviour with traditional methods such as mathematical methods. Therefore, there is always need to model this nonlinear and time-varying system with artificial intelligence techniques to obtain a better accuracy to evaluate the behaviour of EAFs and maintain the quality of power.

\subsection{Objective}

The purpose of this study is to model the EAF with LS-SVM, which is useful for nonlinear systems with time-variant parameters. There are several known approaches for modeling and predicting the behaviour of EAFs. A number of modeling was based on explicit mathematical equations, which are sometimes complicated models for nonlinear and time varying systems. Thus, there is a need to simplify them and sometimes this simplification in the model decreases the model accuracy. Therefore, the data-driven models (DDM) have been used to decrease the complexity of the model and also increase the model accuracy.

Several approaches to data-driven models have been proposed to model EAFs, such as artificial neural networks (ANN), fuzzy logic systems, and neuro-fuzzy, etc. Most of them have been used machine learning techniques to classify the data or predict the arc voltage based on previous information and some of them have been tried to predict the arc current based on the previous data. This study is the continuation of such data-driven approach models by expanding the remodeling of nonlinear $\mathrm{v}-\mathrm{i}$ characteristic in electric arc furnaces [1]. However, in this study we 
have built a model to predict both arc voltage and arc current based on past history of the arc voltage and current by using least square support vector machine (LS-SVM).

\subsection{Proposed Approach}

This thesis proposes a LS-SVM based model to predict the behaviour of the EAF in terms of the $v-i$ characteristics. The main goal of using the LS-SVMs is to take advantage of their learning capability using the actual measurements from the operational furnaces. LS-SVMS are, therefore, very similar in this regard to other learning approaches, mainly neural networks, but they also provided added advantages including avoiding local minima and guaranteed convergence.

\subsection{Thesis Structure}

This thesis is organized as follows. Chapter 2 provides related background including the structure and principal of EAF operation, a literature review of the relevant earlier work, in particular, a review of mathematical and data-driven methods for modeling EAF. Chapter 3 discusses how the support vector machine (SVM) and least square support vector machine (LS SVM) architectures can be applied to model the electric arc furnace and to predict the behaviour

of the EAF. Chapter 4 explains the experimental design. Finally, chapter 5 summarizes the results and provides discussion related to future direction. 


\section{Chapter 2}

\section{Literature Review}

\subsection{Introduction}

In this chapter the structure and principal of EAF operation have been studied. A thorough review of the state-of-the-art modeling approached used for EAFs are provided here. These include mathematical methods and the data-driven methods with particular attention to the machine learning approaches and Support Vector Machines (SVM).

\subsection{Structure and principal of EAF operation}

Electric arc furnaces (EAFs) are one of the primary devices used in the production of steel from scrap and direct reduced iron (DRI). The popularity of EAFs stem from their proven promises in increasing the productivity, and at the same time decreasing both the waste of energy and the operational costs [8]. The operating cycle for EAF, known as the tap-to-tap cycle, has the following operations [9]:

- Furnace charging

- Melting

- Refining

- De-slagging 


\section{- Tapping}

- Furnace turn-around

The first step in EAF operation is loading scrap steel into the furnace from an overhead crane and the scrap falls into the furnace. Then a lid is flapped over the furnace and preheated with burners. The lid holds electrodes which are descended into the furnace and energized. After current is crossed the electrodes, an arc is formed and the heat generated by melting the scrap. The melting is an important part in the EAF operation, and recent designs are attempted to maximize the melting capacity of the EAF. The energy that is used in melting can be electrical or chemical. The melting process is continued until the last scrap is added to the furnace. Refining operations have applied the removal of phosphorus, sulfur, aluminum, silicon, manganese and carbon from the steel. The next operation after refining is de-slagging, that is, the purging of the materials by removing the undesirable materials from the furnace. After purging, the melted steel is spilled into a ladle. Furnace turn-around is the next step that complete the tapping until the furnace is ready for the next heat [9].

Structurally, an electric arc furnace (Fig. 1.1) is made up of a cylindrical chamber which is open at the top, and has an adaptable domed roof, and three graphite electrodes with their associated equipment such as electrode arms, electrode holders, electrode masts, flexible cables, etc.

It is necessary to control EAF at the highest possible average power. The power levels for EAFs are from $10 \mathrm{MW}$ to $100 \mathrm{MV}$. Accurate and dynamic models of EAFs are hence needed to have optimized power control and maintain the quality of power.

A number of difference techniques have already been used to model electric arc furnaces including mathematical approaches, $v-i$ characteristic based models, system identification 
methods, power based models, current/voltage source based models, frequency domain models, stochastic approaches and adaptive approaches [8, 10-48]. We will review these modeling approaches in the next section.

\subsection{Methods for modeling EAF}

In general, we may group EAF modeling approaches into two categories in two. These are (i) mathematical methods, and (ii) data-driven methods. The mathematical methods may include differential equations based approaches as well as probabilities/stochastic algorithms. The datadriven methods (DDMs) are based on computational intelligence and machine learning approached such as neural networks and fuzzy systems. Although the mathematical methods have been used widely for modeling the EAF, recently the data-driven models have attracted more attention.

\subsubsection{Mathematical Models}

The mathematical models are based on nonlinear differential equations, symbolic dynamic, wavelet analysis, Fast Fourier Transform (FFT), probability approaches, $v-i$ characteristic, frequency domain, current/voltage source, and stochastic approaches[8, 10-48]. In the following section, the most common mathematical methods for modeling the EAFs are summarized.

Nonlinear differential equations have extensively been used to model electric arc furnaces. In [23], non-linear differential equations have been used to describe the dynamic arc models. This model presented a set of routines for power system harmonic applications. The nonlinear characteristics were explained by using polynomials for fitting of the characteristics or applying 
a Fast Fourier Transform. The proposed model could be used for arc discharge lamps as well as arc furnaces. In [45], the Newton algorithm was used for an iterative time and frequency domain simulations. Frequency domain included all linear parts and time domain included nonlinear and time-varying components. Three methods of identification were used in the study. These methods were matrix exponential, direct approach, and numerical differentiation. Since the proposed hybrid methodology was general, it could be applied to the periodic steady state solution of the three-phase system [45]. In [25], two Newton based methods have been used to model the dynamic behaviour of nonlinear and time-varying components. The Newton techniques were based on the direct approach and the numerical differentiation procedures. Saving energy was the principal in this study, which was same as the model that presented in [23]. In this work two methods were applied for the acceleration of time domain computations. One was the direct approach method (DA), and the other one was the numerical differentiation method (ND). This ND method was obtained from the previous work in [45]. There is another mathematical model of the AC EAF based on bifurcation theory which is a set of ordinary differential equations[49]. In this work, a general dynamic arc model was rely upon the conservation of energy, which was same in [23]. The differential equation model was based on the conservation of energy. The authors believed that the application of bifurcation theory to other models was feasible [49].

Another mathematical method that has used to model the EAF is based on symbolic dynamic and wavelet analysis [29]. The structure of the symbolic dynamics method is to code the signal in symbols, so the levels of the signal can be separated. Symbolic dynamic was applied to obtain a correction current to characterize a highly varying load current for an electric power load [29]. There are several models based on wavelet analysis. One of them used Wavelet transform which is suitable for transients and harmonic analysis [11]. To verify the effectiveness of the 
application, an actual arc furnace was used as an example. This method was suitable for linear systems because impedance or admittance matrix methods were used instead of forming the state equations. However, the disadvantage of this method appears when required resolution level is high or the system dimension is large [11]. Another application used the Morlet wavelet function to study voltage flicker-generated signals [46]. The Morlet wavelet was applied as a basic function for the continuous wavelet transform (CWT). After several test scenarios, it was found that this method was effective in analyzing those flicker-generated signals. The wavelet method applied to investigate the voltage flicker of the EAF, because the EAFs are major cause of voltage fluctuation in electrical networks. Although the proposed method had feasibility in power quality problems, but it did not offer a reasonable wavelet transform for the EAF [46]. In [47], a continuous wavelet transform model was described to measure voltage flicker by using Gaussian Wavelet function. In this study, the experimental results were compared with those from the method reported in [46]. It was found that this method has higher reliability and visualization. The author suggested that the implementation of the proposed method can be improved [47].

There are several mathematical methods that use FFT to model the EAFs. For instance a method has been reported that it had a good estimate for the flicker of dc and ac arc furnaces. This method has provided suitable results. However, the results were not good for measuring flicker. The IEC flicker meter must be studied as measurement system in this method [37]. Another $v-i$ prediction approach has been introduced by using Markov chain processes, which were derived from the fast Fourier transform (FFT) frequency decomposition method. Both accuracy and efficiency have been improved by using this method. The accuracy could be improved by increasing the number of harmonics, but it has computational complexity [31]. 
Some mathematical models rely upon probability approaches. Two probability based methods have been discussed to simulate arc furnace flicker [21]. A probability approach and Gaussian distribution have been used to model arc voltage and arc resistance for study and simulation of arc furnace flicker [21]. Another work [50], has introduced a statistical model for predicting arc furnace flicker emission by measurements on practical systems. The system modeled the correlation between parameter changes and variations in flicker level to have higher production and lower flicker. The authors suggested that the proposed method could be applied to consider the effect of system parameter variations on flicker fluctuation. In another study [51], a threephase arc model for systems with low-voltage AC was proposed using a probability approach based on characteristics of three-phase fault arcs. The minimum three-phase arcing fault current in systems with low-voltage AC could be obtained with the proposed method or by solving the differential equations.

Another mathematical group of studies are based on $v-i$ characteristics to model the electrical arc furnace. In [52], a time domain controlled voltage source (CVS) model has been developed for an arc furnace by using the Electro Magnetic Transients Program (EMTP). This model performed not only static (deterministic) but also dynamic arc characteristics. Static arc models were used in harmonic studies, and dynamic arc models were used in power quality and voltage flicker studies. The results showed that the active power consumed by the arc furnace load was significant in the process of modeling the arc furnace. another arc furnace model has been reported with the stochastic nature of the electric arc current/voltage characteristic and three phase auto regressive model [53]. Voltage fluctuation simulation in this model did not completely represent the high non-linearity of the EAF. Also the nonlinearities in the transformer 
were not modeled in this simulation. Therefore, the proposed model could be improved by solving these simulation issues [53].

Another frequency domain approach has been introduced to model EAFs based on a dynamic and nonlinear $v-i$ characteristic of the electric arc and also continuous evolution of the arc current [30]. Newton's method has been applied in this model to determine the equations for harmonic balance and current zero crossing of the model. As a result, it was found that the capacitors bank could modify the harmonic emission and inter-harmonic spectrum. However, the proposed model needed to improve the characterization of the EAF [30]. A v-i characteristic based method has been presented to model an adaptive arc furnace by using a current-controlled nonlinear resistance [26]. This model was able to control the arc furnace load, which made it suitable for different operating conditions. The proposed model could be adaptively adjusted to obtain the given operation condition. This model was efficient in the power quality study [26].

A time domain based model has been reported which is based on the $v-i$ characteristic of the arc, especially for the harmonic analysis of EAF. This model is implanted in EMTDC [20]. The proposed model simulated the three-phase arc furnace with harmonics perspective [20]. In the previous studied, stochastic ideas were used to obtain the nonlinear and time-varying behaviour of the EAF [52]. So, another arc furnace model was presented by using chaotic and deterministic elements for harmonic analysis. This model is implemented in a Simulink environment. The model obtained multivalued $v-i$ characteristics by dealing with the corresponding differential equation, which were presented in previous work [23]. Furthermore, an IEC flicker-meter was implemented for evaluating the sternness of fluctuation in the arc furnace voltage simulation [44]. In [54], a power based method has been developed for measuring the voltage fluctuation of the electric arc furnace by using both the reactive and active power variations. The maximum 
apparent power fluctuation method (MAPFM) has been proposed in this model. This method reduced the variations between the predicted voltage flicker and the actual value from EAF operation.

There are several mathematical models that use the current or voltage source in the time domain. A model has been introduced in [55] that has been used a harmonic voltage source, field measurements, and filter design work in a steel manufacturing facility. It was recommended that the power quality and plant productivity should be improved in steel manufacturing facilities by extending the power factor and decreasing the amount of harmonic distortion. In [41], a frequency domain based model for EAF has been presented for iterative harmonic analysis (IHA) by using the Newton Method. The model has been used to investigate arc current for both single and three-phase structures [41]. Another method has been developed to model the arc currents in an electric arc furnace which has been used nonlinear analysis and chaos theory [43]. The model was examined by applying the actual current data from an EAF. The results showed that the model did not have a reasonable accuracy. However, it could be used for power system planners as a useful device for simulation. Although the model could predict the general behaviour of the load current, it could not provide an accurate prediction of exact current level [43].

A number of current-voltage source models have been presented to model the EAFs. For instance, an EMTP based arc furnace model has been reported to model the EAF, which it has been used a generator for voltage in the time of melting operation. The purpose of this model was to reduce voltage flicker in arc furnaces [56]. The model has obtained a reasonable explanation of the arc furnace procedure. However, the estimations of flicker were not accurate enough [56]. Another method modeled the arc furnace that included non-linear time varying 
resistance. Two time-variation laws of arc length have been used in this method. One of the laws was a periodic, sinusoidal law and the other one was a band-limited white-noise law. The model has been implemented by EMTP. As a result, the accuracy could be improved by using threephase simulations [42]. Therefore, another model for the arc furnace has been reported by same authors to simulate cases from the primitive model of the arc furnace, which simulated both voltage and current waveforms and arc characteristics. This model has been implemented by the EMTP which used a sinusoidal or white-noise law. This simulated model, which was based on generators for harmonic voltage, has not been obtained the satisfied accuracy for designing [57]. Another simulation model has been implemented in [53] to represent the voltage disturbances for voltage fluctuations and harmonics. The proposed model focused on the alteration of the electric arc voltage. The model has been used a stochastic nature of electric arc current-voltage characteristics. The time variation of the arc length has been modeled by using white noise and AR and ARX. The simulation results showed the measured voltage fluctuation and harmonic currents accurately. However, the authors offered to improve the simulation by using nonlinearity in the transformer [53].

Another group of mathematical methods is based on frequency domain. An analog model has been applied in [34], which can be used for the steady-state harmonic analysis of the electric arc furnace load. The elementary step in simulation was to obtain the analysing a feedback of frequency on the power network. The harmonic currents on a system could be predicted by using the proposed model. However, the authors suggested that the consequences of employing power factor correction capacitors needed to be studied in order to improve the model [34]. Another model has been reported, which is based on an interval-wise constant arc voltage. The model was applied for simulation the time-varying reactive power demand, flicker, and harmonics [18]. Another mathematical model has been presented in [14], which used time and frequency domain 
analysis technique to determine voltage flicker. The proposed algorithm was fast and simple. However, the model had limitations in the simple models of low order because of the lack of specific data [14]. Later, another frequency domain model has been reported to drive the analytical expressions for study of the instantaneous flicker. The model employed to the voltages of both dc and ac arc furnaces on the standard IEC flicker meter. The proposed method had a reasonable estimate for flicker of arc furnaces. Although the model was suitable as a different approach for predicting flicker, but it was not suitable for calculating flicker [37].

Several mathematical methods have been developed to model the behaviour of the EAF that are based on stochastic approaches such as Markov model, stochastic white noise, random process methods, and chaotic attractor to model the EAFs. In [40], a model has been presented to predict flicker by using a band limited white noise single-phase flicker generating source with a magnitude. The proposed model was based on stochastic assumption for the time-varying law. Another arc furnace model has been reported [26], which is based on the white-noise law, to estimate flicker in EAFs. The approximations in the proposed model were more effective than those used in [40]. Although the model was effective in flicker-compensation systems, its accuracy could be improved by using three-phase simulations [26]. Another dynamic model has been presented to predict voltage flicker for ac arc furnaces by applying the band limited white noise and the equivalent arc resistance [39]. A variable arc length, which was explained in the model [40], was used to characterize. In the same time, another arc furnace model has been applied the stochastic nature of the electric arc current-voltage characteristics [16]. This model was used for voltage fluctuations and harmonics. The short-term flicker severity index, which was very close to the value presented in [26], has been used in the simulation tool to analyze the behaviour of an arc furnace as a source of disturbance. The result of this study was discussed in previous part. 
A chaotic dynamics method has been presented in [43], which was the first model in chaotic dynamics, for providing the operation of nonlinear loads in power systems such as EAF. In [58], a stochastic voltage-flicker power flow model has been developed to analyze the impact of voltage flicker sources, which is very applicable method. The instantaneous arc voltages in proposed model have been applied from [26], [16]. However, another model has been reported to study harmonic of the arc furnace that is based on the chaotic and deterministic elements. The model has been implemented in Simulink [44]. Unlike previous studies that had difficulty to capture the even harmonics, this model was able to capture even harmonics. In [31], another model described the $v-i$ prediction approach for the electric arc furnace based on Markov chain processes. The model has been implemented by using an active harmonic filter (AHF) in Simulink. The accuracy of the proposed model could improve by increasing the number of harmonics. Another chaotic approach has been reported in [59], which was based on three chaotic attractors (Rössler, Chua [44], and Lorenz attractors [43]) for the DC arc. Both tuned Chua model and the tuned Lorenz model had a good performance in wave form distortion evaluation, but less efficient performance in voltage fluctuation evaluation. As a result, authors suggested that the model could be tuned for better prediction of voltage fluctuation [59].

Full mathematical modeling is desirable, but it is sometimes not accurate or flexible enough to use for modeling the EAF. The high complexities of the mathematical models make it difficult to process them correctly. Also these models are particularly complicated models for nonlinear and time-varying systems. To make these models easier and reasonable to use for modeling the nonlinear systems, simplifications are necessary. However, the model simplifications decrease the model accuracy. Therefore, there is a need to use a model that can improve the accuracy and reduce the complexity of the mathematical models. So the data-driven models (DDM) were used for modeling the EAF. The data-driven models are more objective, less experience-based and 
more scientific models. These models are based on real system data and are useful, reliable and not complex with better performance and reasonable accuracy.

\subsubsection{Data-Driven Models (DDM)}

Data-driven models have the ability to model the nonlinear systems where a mathematical representation cannot be found or is ill-defined. In last decade, several data-driven approaches have been proposed to model the EAF. There are several areas contributing to DDM such as data mining, computational intelligence, and machine learning. In this section, the machine learning techniques for modeling EAF are presented. Data-driven modeling (DDM) relies upon the analysis of the data characterising of the EAF and also it is based on connections between the variables in a system such as input and output variables. A few assumptions about the "mathematical" behaviour of the EAF were used in DDM.

One of the most popular machine learning approaches to model the EAF is artificial neural networks (ANNs). A feed-forward neural network was described in [60] to model arc furnace electrical wave forms. Data were obtained from an experimental EAF to predict the state of EAFs. The authors suggested that the model could be improved by adding more hidden layer neurons. Another neural network based method was reported for modeling the EAF in [3], where two different architectures, the Multi-Layer Perceptron (MLP) and the Radial Basis Function (RBF), were discussed. The RBF model simulated the nonlinear behaviour of EAF successfully and it had a reliable accuracy.

Chaos theory and neural networks have also been applied to model the DC EAF [35]. The radial basis function based model [2] was used to train and learn the model dynamics Due to the fast capability of learning and ability of classification for radial base function network (RBFN), it 
was employed to predict the arc voltage signal. This model had a reasonable accuracy. However, when the model was simulated in EMTP (a widely used software to simulate the power systems), several problems still remained in the model. The authors offered that these problems could be solved by increasing the performance of heat transfer and reducing the negative consequences of DC arc with controlling the arc furnace state [35]. Two models have applied RBF inverse identification neural networks for electrode system in the EAF [61], [62]. These models were based on nearest neighbor clustering algorithm with adaptive parameter. The system was used in third-iron-steel plant of Ma'anshan China. An improved model based on [61] was reported to have a powerful real time ability and also it can work as a reliable PLC control system [62].

Another neural network method with a standard back-propagation (BP) algorithm has been used for modeling the EAF, but the BP algorithm had negative impacts on the model. Therefore, another NN with an improved BP algorithm has been applied by using adaptive variable step size. The second model had better accuracy and faster convergence speed [63]. Later, another model using a combined RBFNN method and the lookup table (LUT) method has been used to predict voltage-current waveforms in time-varying EAF [11]. After comparison the simulation of this combined model with those achieved from regular RBFNN/BPN models, it was found that this combined method can model the $v-i$ characteristic of evaluating the waveform distortions as well as flickers, and reactive power consumptions of the EAF successfully [11].

In [64], an ANN has also been applied to predict the reactive power of EAFs to achieve a better Static VAr Compensator (SVC) performance and reduce the flicker by using 20 neurons in the hidden layer of ANN. The data for the model were gathered from eight operational arc furnaces in Iran. This model was based on concepts of flicker frequencies and power spectral density (PSD). It improved the SVC performance in prediction of EAF reactive power. 
Another machine learning approach to model the EAF is fuzzy logic system. An application of adaptive fuzzy logic systems was reported in [3], which used the same data in RBF model [2], to model the electric arc furnace. This method was based on three properties of logic systems such as nonlinear black-box modeling and universal approximation and also the functional equivalence with RBF network. Since classic fuzzy identification method used the trial-and-error style for training, the accuracy of this fuzzy logic system (FLS) was more effective and also this model had faster training time than RBF model [3]. In [65], both classic and adaptive fuzzy logic systems (FLSs) have been used to model the behavior of the EAF. Both techniques have been described as nonlinear black-box modeling techniques. After several implementations, it has been shown that the adaptive fuzzy logic system has faster training time and the results compare well with actual data from the EAFs [65].

[17] reports an application of a direct adaptive output-feedback fuzzy control was used to model the three-phase arc furnace electrode regulator system by dividing the system in to three subsystems. This model was applied the Lyapunov function in the fuzzy ruler's parameters. The model obtained good control performance, had reliable dynamic and static characteristics, and was able to keep the external disturbances effectively.

Several researchers have used hybrid machine learning algorithms such as neuro-fuzzy to model the EAF. For example, A recurrent neuro-fuzzy predictors was reported for multi-step prediction of $v-i$ characteristics of EAFs in [66]. This recurrent predictor applied fuzzy rule-base by employing data clustering techniques and arranged the parameters of these rules by using neural network. The proposed model was suitable for long-term prediction for both current and voltage of EAFs. Also, this model was proved to be capable of obtaining the complex, nonlinear characteristics of EAFs [66]. A similar feed-forward neuro-fuzzy network has also been applied 
to predict the $v-i$ characteristics of EAF [67]. It was demonstrated that the feed-forward neurofuzzy model was not only improved the quality of multi-step prediction but also was practical for long-term prediction. The structure was used in this model was different from the recurrent model [66]. Recurrent predictor network had a multi-step-prediction serial structure where the predicted outputs in multi-step prediction could be repeatedly provide back to the system input and applied to calculate the next outputs [66]. However, the second model used multi-step prediction process that was consist of number of independent and parallel single-step prediction operations [67]. The proposed predictor was capable for accurate multi-step prediction and its performance increased compared with the previous model.

Another application of two classes of neuro-fuzzy networks was presented in [68]. Both feedback and recurrent networks were employed to predict the current and voltage of EAFs. These networks applied the fuzzy-rule-base by using data clustering and adjust the parameters of the rules by using neural network. Several predictors were implemented with different training parameters. After assessment of the predicted data and the real data, it was found that the model had reliable performance and was capable of predicting the complex and nonlinear characteristics of EAFs [68]. Another adaptive neuro-fuzzy inference system (ANFIS) modeled a regulator control loop of the electric arc furnace, which manages the setting of the electrodes in EAF as a non-parametric method. The data in this model were collected from the EAF of the Gerdau Ameristeel Whitby (GAW) in Ontario, Canada. This model needed to improve the modeling by applying an adaptive learning for the entire system to have more effective results in simulation [15].

An AC EAF model based on neuro-fuzzy networks was reported in [69]. The results indicated that the proposed model not only had high control accuracy but also had improvement in having 
an accurate speed of the electrodes moving up and down. Later another neuro-fuzzy networks based model was reported for online estimation of EAF tap temperature [70]. This model used neural networks as a classifier and fuzzy inference function as a predictor for a temperature. The proposed model decreased the errors in temperature prediction. On-line estimation of tap temperature helped optimization of the EAF process [70].

An adaptive ANFIS based was proposed to simulate the EAF by using data from Gerdau Ameristeel Whitby (GAW) in Ontario, Canada. The focus of the proposed model was on the regulator loop or positioning of the electrodes. This model simulated the regulator loop to predict active current, reactive current, voltage, and regulator output voltage. However, this system was not complete, and it needed to simulate the entire EAF system [41]. Another ANFIS model was reported to dynamically reconstruct the nonlinear $v$ - $i$ characteristic in EAF[1]. The data applied for training this model are the real measurements with a selection frequency of $1920 \mathrm{~Hz}$ from the EAF process. Fuzzy rule-based system was used to model the EAF by applying different sets of training/validation data, but the results for this method was not successful. So, the ANFIS were used for EAF modeling. This model can optimize the fuzzy rules from training data. After comparison of these two models, which used the exact same data set, the result was a reduction in the number of rules and significant enhancement in performance error. This application has predicted the arc voltage based on the past history of the arc voltage and current, as well as the current derivative values [1]. In this thesis, we have investigated the proposed model to predict arc voltage and current by using least-square support vector machine. 


\section{Chapter 3}

\section{Methodology}

\subsection{Machine Learning}

Machine learning, a branch of artificial intelligence (AI), is the science of enabling computers to learn and to adapt to situations to which they have not been exposed before. In particular, machine learning provides computers the capability of learning with or without being programmed [71]. The purpose is to design a machine that does the learning automatically without human assistance. There are two general types of learning algorithms:

1) Supervised Learning: The observations are labeled. The inputs and outputs are given and the task is to classify data by supervising the data,

2) Unsupervised Learning: The measurements are not labeled, which is meant that the target variable is unknown. The goal is to find the hidden structure in observations.

The difference between supervised and unsupervised learning is in the structure of the model. In supervised learning, the system learns a mapping between inputs and outputs to predict new values. However, unsupervised learning is learning without a training set.

The data are usually divided between a training set and test set. The algorithm extracts knowledge from the training set and builds the model. After the model has been built by using 
the training set, then the model can be confirmed by making predictions against the test set. The purpose of the test set is to assess the performance of the algorithm. Both methods are powerful and valuable, but choosing one of them is depend on the kind of the problem and times to solve this problem. Supervised learning is divided in to two broad categories:

- Classification

○ Binary Classification: the output has two values, such as true and false.

- Multi-class Classification: the output is label

- Regression: the output is real value.

\subsection{SVM}

\subsubsection{Classification and Regression}

There are many different types of classifiers in machine learning including support vector machines (SVM). SVMs belong to a family of generalized linear classifiers. Based on various studies, SVMs have greater performance in terms of classification accuracy than the other data classification algorithms [72].

SVMs have been applied in several of real world problems such as hand-written digit recognition, text categorization, tone recognition, image classification, data classification and object detection, pattern classification, and diseases prediction. In many problems, SVMs perform much better than other non-linear classification such as artificial neural networks and knearest neighbors. In this section, a literature review on SVM is summarized. 
A support vector network used in [73] as a two-group classification problems. Several classical algorithms such as linear classifiers, k-nearest neighbors classifiers, and neural networks were tested. These methods were applied to two experiments with different databases. The results showed that the SVM has a more reliable performance than other methods.

In [74], SVM was used in phonetic classification. Three databases were used in this experiment. Two databases were applied for vowel classification and the other database was used in phonetic classification. In these classifications both a polynomial kernel and RBF kernel were used and the results showed that the SVM performance was better than neural networks and Gaussian classifiers. In [75], SVM used for speech recognition system as a classifier. The application has a reasonable improvement in error rate on a continuous alpha digit task and a speech task and also it has a noteworthy performance in classification.

In [76], A hybrid neural network based on SVM was proposed for credit rating analysis and prediction by using two datasets of United Sates and Taiwan markets. After comparison two methods, it was found that the accuracy that SVM achieved is comparable to the BNN, which is around $80 \%$.

In [77], a survey was presented to monitor the machine condition and fault diagnosis by using SVM. An application was presented in [78], to predict travel-time in the intelligent transportation system and traveler information system. Support vector regression (SVR), typically used for time-series analysis and forecasting as well as statistical learning, was used to predict accurate travel-time for this application. The results of comparing this model with other methods showed that SVR predictor reduced both relative mean errors (RME) and root-mean-squared errors 
(RMSE). Another application used SVM to predict bus arrival time [79]. One of the major elements in transit operation is to have a model to predict the time for transit vehicle arrival with suitable accuracy with consideration of traffic conditions. The results of comparison between the ANN and SVM showed that the SVM presented excellent indication of the bus arrival time between two time points. Although the ANN models are the local optimal solutions for bus-time arrival, but the SVM may be globally optical. The SVM is a dominant solution for bus arrival time prediction.

Classification is one of the most main problems in machine learning. There are a lot of methods in learning machines that we can use for classification, such as Neural Networks, Naïve Bayes, and Support Vector Machines, Nearest Neighbor, and decision trees.

Since prediction over two classes is the most common type of prediction in real-world problems and SVM provide accurate binary classification, most scientists use SVM in classification. This method was introduces by Cotes \& Vapnik in 1995 [4]. The binary classification problem is a learning problem with binary outputs. The problem for real-valued outputs is known as regression. Classification is attained by a linear or nonlinear separating surface in the input space of the dataset [72].

Classical learning methods such as neural networks are used to minimize error on the training data set, and it is called empirical risk minimization (ERM). However the SVM is based on structural risk minimization (SRM). SRM is gained from a minimization of the upper bound of generalization error $[22,73,80]$. Since the SRM is minimized in the SVM classifier, the classifiers that are based on SVM have efficient generalization properties in comparison to other classifiers [72]. 
Assume that we have $\boldsymbol{x}_{\boldsymbol{i}}(i=1,2 \ldots M)$ as an input vector, $M$ is the number of observations. Each observation includes $\left(\boldsymbol{x}_{i}, \boldsymbol{y}_{i}\right)$ where $\boldsymbol{y}_{i}$ is a label with a value of -1 or 1 as an output vector. SVM attempts to plot a hyperplane in space which can divide all classes in the most efficient way. We can show this hyperplane for linear data with:

$F(x)=\boldsymbol{w}^{\mathrm{T}} x+b=0$

$F(x)=\sum \boldsymbol{w}_{i} x_{i}+b=0$

where $\boldsymbol{w}$ is M-dimensional vector and $b$ is a scalar. Both $\boldsymbol{w}$ and $b$ are used to present the position of the hyperplane. The decision function to create the separating hyperplane is a sign $f(x)$ which separates input data in to either a positive class or negative class[72].

$F\left(x_{i}\right)=1 \quad$ if $\quad y_{i}=1$

$F\left(x_{i}\right)=-1 \quad$ if $\quad y_{i}=-1$

or in a complete equation

$y_{i} F\left(x_{i}\right)=y_{i}\left(w^{T} x_{i}+b\right) \geq 1 \quad$ for $i=1,2, \ldots, \mathrm{M}$

The best separating hyperplane is the one that creates the maximum distance between the plane and nearest data [72]. In Fig. 3.1, an example of the ideal hyperplane of two data sets is shown. Two different classes of data are presented, squares for negative class and circles for positive class. The SVM tried to align a linear margin between the two different classes and also tried to provide that the distance between the boundary and the nearest data point in each class is maximal [72]. The margin is the place between these nearest data points in two classes and the 
boundary is placed in the middle of this margin. The nearest data points are called support vectors which shown by blue squares and red circles. These support vectors are used to define the classifier.

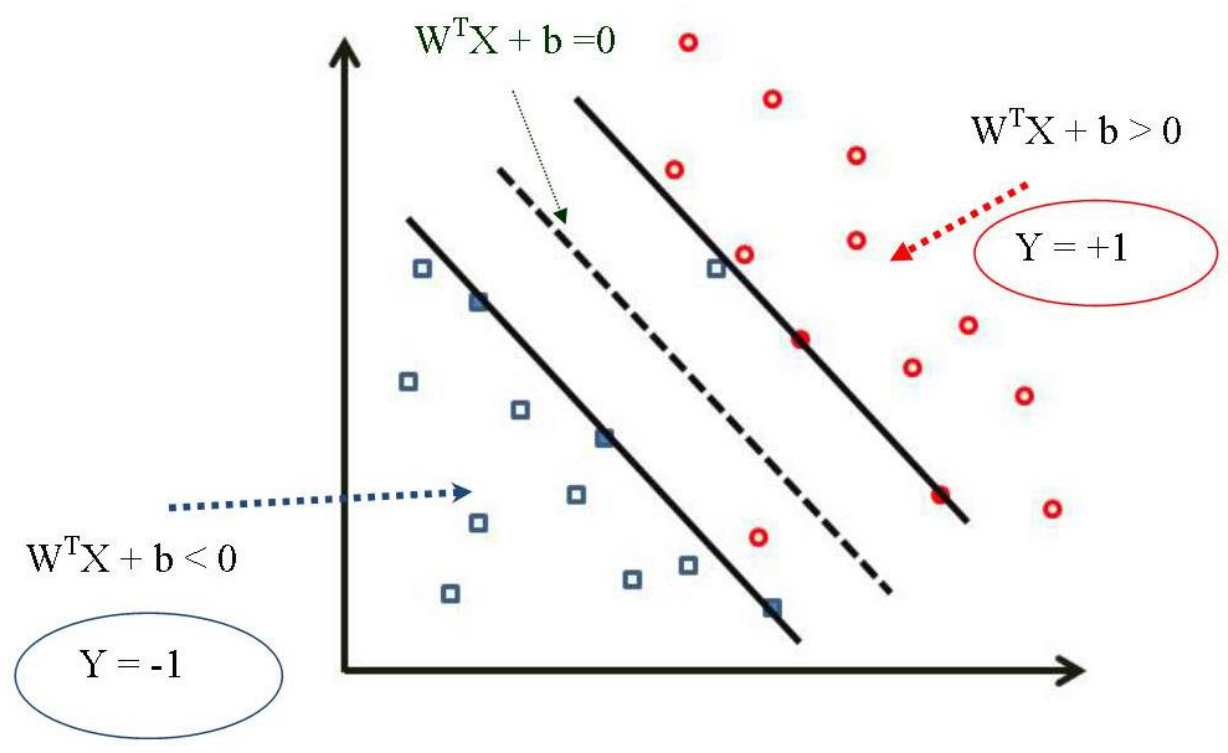

Fig. 3.1. Optimal Hyperplane of two data set

The distance of the closest data points is:

$d(w, b)=\min \frac{w x_{i}+b}{|w|}-\max \frac{w x_{i}+b}{|w|}$

From (3.3) and (3.4), we conclude that the minimum and maximum values are +1 and -1 . So the distance of the closest points is:

$d(w, b)=\frac{1}{|w|}-\frac{-1}{|w|}=\frac{2}{|w|}$ 
The problem is maximizing $\frac{2}{W}$, which is equivalent to minimize $\frac{|w|^{2}}{2}$. The Lagrange multiplier applies to the function to calculate the dot product between vectors and to simplify the formula as:

$d(w, b) L(\vec{w}, b, \alpha)=\frac{1}{2}\|w\|^{2}-\sum_{i=1}^{N} \alpha_{i}\left[y_{i}\left(\vec{w}^{T} \vec{x}_{i}+b\right)-1\right]=\frac{1}{|w|}-\frac{-1}{|w|}=\frac{2}{|w|}$

Minimizing $L(w, b, \propto)$ with respect to $\mathrm{w}$ and $\mathrm{b}$.

$$
\begin{gathered}
\frac{\partial}{\partial w} L(\vec{w}, b, \alpha)=0 \Rightarrow w=\sum_{i=1}^{N} \alpha_{i} \vec{x}_{i} y_{i} \\
\frac{\partial}{\partial b} L(\vec{w}, b, \alpha)=0 \Rightarrow \sum_{i=1}^{N} \alpha_{i} y_{i}=0
\end{gathered}
$$

a) Maximizing $L(w, b, \propto)$ with respect to $\alpha_{i}$

$$
\begin{aligned}
L(\vec{w}, b, \alpha)=\frac{1}{2} & \|w\|^{2}-\sum_{i=1}^{N} \alpha_{i}\left[y_{i}\left(\vec{w}^{T} \vec{x}_{i}+b\right)-1\right] \\
& =\frac{1}{2} \vec{w}^{T} w-\sum_{i=1}^{N} \alpha_{i} \vec{w}^{T} \vec{x}_{i} y_{i}-b \sum_{i=1}^{N} \alpha_{i} y_{i}+\sum_{i=1}^{N} \alpha_{i}
\end{aligned}
$$

b)

$$
\begin{aligned}
& L(\vec{w}, b, \alpha) \Rightarrow Q(\alpha)=\sum_{i=1}^{N} \alpha_{i}-\frac{1}{2} \sum_{i=1}^{N} \sum_{j=1}^{N} \alpha_{i} \alpha_{j} y_{i} y_{j} \vec{x}^{T}{ }_{i} \vec{x}_{j} \\
& \sum_{i=1}^{N} \alpha_{i} y_{i}=0 \quad, \alpha_{i} \geq 0 \text { for } i=1, \ldots, N
\end{aligned}
$$


There are quadratic programming (QP) techniques for solving the problem. The answer is explained as following:

$$
w=\sum \alpha_{i} y_{i} x_{i}
$$

and

$b=1-w x_{i}$

Up to now, we assume that the data can be separated with one line. However, the data in real world cannot be separated easily because they have noise and errors. Therefore, slack variables are used to measure the deviation of data point from the best separation. The new formula with slack variables is as following:

$$
\phi(\vec{w}, \xi)=\frac{1}{2}\|\mathrm{w}\|^{2}+C \sum_{i=1}^{\mathrm{N}} \xi_{\mathrm{i}}
$$

where

$y_{\mathrm{i}}\left(\mathrm{w} \cdot \mathrm{x}_{\mathrm{i}}+\mathrm{b}\right) \geq 1-\xi_{i}$

The $C \sum \xi_{i}$ is showed the maximum number of errors. The $C$ parameter, which is used to control the overflow, is regularization and it should be selected by user.

Maximize $Q(\alpha)$ :

$Q(\alpha)=\sum_{i=1}^{N} \alpha_{i}-\frac{1}{2} \sum_{i=1}^{N} \sum_{j=1}^{N} \alpha_{i} \alpha_{j} y_{i} y_{j} \vec{x}^{T}{ }_{i} \vec{x}_{j}$

Subject to:

$$
\sum_{i=1}^{N} \alpha_{i} y_{i}=0 \quad, 0 \leq \alpha_{i} \leq C \text { for } i=1, \ldots, N
$$


then

$\left\{\alpha_{i}\right\} \Rightarrow w=\sum \alpha_{i} y_{i} x_{i}, b=y_{k}\left(1-\xi_{k}\right)-w^{T} x_{k}$

$\Rightarrow f(x)=\sum \alpha_{i} y_{i} x_{i}^{T} x+b$

If data is not linearly separable, then original data can be transformed into the new space by using kernel function.

\subsubsection{Kernel function}

Kernel function is a function that based on the dot product and can be written as:

$k\left(x_{i}, x_{j}\right)=x_{i}^{T} x_{j}$

The kernel function is used to map non-linear input data in to a higher dimensional feature space.

The nonlinear vector function $\varphi(\mathrm{x})=\left(\varphi_{1}(\mathrm{x}), \ldots, \varphi_{\mathrm{n}}(\mathrm{x})\right)^{\mathrm{T}}$ map the input data to feature space as shown in Fig. 3.2 When the input data transfer with $\varphi: x \rightarrow \varphi(x)$ to higher dimensional feature space, the kernel function is as following:

$k\left(x_{i}, x_{j}\right)=\varphi\left(x_{i}\right)^{T} \cdot \varphi\left(x_{j}\right)$

Some widely used kernel functions are

$\left(x^{T} x^{\prime}+1\right)^{d} \quad: \quad$ Polynomia 
$\exp ^{\left(-\gamma|| x-x^{\prime} \|^{2}\right)} \quad: \quad \mathrm{RBF}$

$\tanh \left(x^{T} x^{\prime}+t\right) \quad: \quad$ Sigmoid

In the first function, with $d=1$ function is the linear kernel. The second kernel is the Gaussian kernel function, where $\gamma>0$ is a parameter that defines the width of Gaussian.

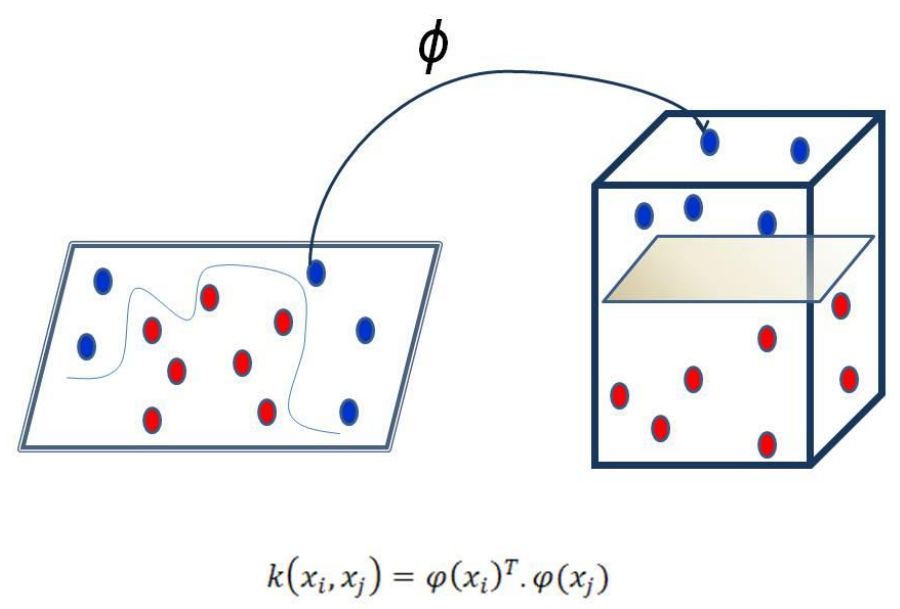

Fig. 3.2. Mapping in Higher Dimensions

\subsection{LS-SVM}

The least squares formulation of SVM, called LS-SVM, which is useful for explaining linear KKT (Karush-Kuhn-Tucker theorem) systems [81]. The difference between SVM and LS-SVM is solving linear systems instead of QP problem. LS-SVM is a modified version of SVM, which works like a prediction tool for classification and regression for the large data. Suykens and Vandewalle in 1999, have changed Vapnik's standard SVM into the following formulation [82]: 
$F(x)=w^{T} \varphi\left(x_{i}\right)+b$

where $\varphi($ ) maps the input data into a higher dimensional feature space and $w$ is a weight vector and $\mathrm{b}$ is a bias term. In LS-SVM, again assume that the input is a set of examples $\left(x_{i}, y_{i}\right)$ and the purpose is to estimate the function $F(\mathrm{x})$. To estimate the $F(\mathrm{x})$, the following optimization problem is formulated:

$\operatorname{Min} J(w, b, e)=\frac{1}{2} w^{T} w+r \frac{1}{2} \sum e_{i}^{2}$

Subject to $y_{i}$

$F(x)=y_{i}\left[w^{T} \varphi\left(x_{i}\right)+b\right]=1-e_{i} \quad i=1, \ldots, N$

where $N$ is the number of examples, $e_{i}$ is the fitting error.

$L(w, b, \propto)$ with respect to $\alpha_{i}$ ( Lagrange multiplier) in (3.20) can be optimized with conditions in (3.21).

$L(w, b, e ; \alpha)=J(w, b, e)-\sum \alpha_{i}\left\{y_{i}\left[w^{T} \varphi\left(x_{i}\right)+b\right]-1+e_{i}\right.$

Conditions for optimization:

- $\frac{\partial L}{\partial w}=0 \quad w=\sum \alpha_{i} y_{i} \varphi\left(x_{i}\right)$,

- $\frac{\partial L}{\partial b}=0 \quad \sum \alpha_{i} y_{i}=0$,

- $\frac{\partial L}{\partial e_{i}}=0 \quad \alpha_{i}={ }^{\gamma} e_{i} i=1, \ldots, N$,

- $\frac{\partial L}{\partial \alpha_{i}}=0 \quad y_{i}\left[w^{T} \varphi\left(x_{i}\right)\right]-1+e_{i}=0 \quad i=1, \ldots, N$ 
These conditions lead to the following linear system [5]:

$$
\left[\begin{array}{ccc|c}
I & 0 & 0 & -Z^{T} \\
0 & 0 & 0 & -Y^{T} \\
0 & 0 & \gamma^{I} & -I \\
\hline Z & Y & I & 0
\end{array}\right]\left[\begin{array}{c}
w \\
b \\
e \\
\hline \alpha
\end{array}\right]=\left[\begin{array}{c}
0 \\
0 \\
0 \\
\hline \overrightarrow{1}
\end{array}\right]
$$

Where

$\mathrm{Z}=\left[\varphi\left(x_{1}\right)^{T} y_{1} ; \ldots ; \varphi\left(x_{N}\right)^{T} y_{N}\right]$

$$
\begin{aligned}
& \mathrm{Y}=\left[y_{1} ; \ldots ; y_{N}\right], \\
& 1=[1 ; \ldots ; 1], \\
& e=\left[e_{1} ; \ldots ; e_{N}\right], \\
& \alpha=\left[\alpha_{1} ; \ldots ; \alpha_{N}\right]
\end{aligned}
$$

The solution can be written as:

$$
\left[\begin{array}{c|c}
0 & -Y^{T} \\
\hline Y & Z Z^{T}+\gamma I
\end{array}\right]\left[\frac{b}{\alpha}\right]=\left[\begin{array}{c}
0 \\
\hline \overrightarrow{1}
\end{array}\right]
$$

$\Omega=Z Z^{T}$ (Mercer's condition) can be used,

where

$$
\begin{aligned}
\Omega_{i l} & =y_{i} y_{l} \varphi\left(x_{i}\right)^{T} \varphi\left(x_{l}\right) \\
& =y_{i} y_{l} K\left(x_{i}, x_{l}\right)
\end{aligned}
$$


After solving the optimization problem by using Lagrangian function with (3.22) and (3.23), the following equation has been obtained.

$F(x)=\sum_{i=1}^{N} \alpha_{i} K\left(x, x_{i}\right)+b$

Several kernel functions can be used, such as:

- Linear kernel

- Multilayer Perceptron kernel

- Polynomial kernel

- Radial Basis Function kernel

However, the extensively used kernel function is the Gaussian RBF.

$K\left(x, x_{i}\right)=e^{-\frac{\left\|x-x_{i}\right\|^{2}}{2 \delta^{2}}}$

LS-SVMs have successfully been applied in several problems such as breast cancer diagnoses, classification of diabetes disease, financial time series forecasting, prediction of flashover voltage of insulators, application in electrical load forecasting, and modeling a solar air heater [83-88]. 


\section{Chapter 4}

\section{Results and Discussion}

\subsection{Introduction}

This section explains how LS-SVM architectures have been used to model electric arc furnaces. To model the characteristics of the electric arc furnace, the dataset includes the furnace current and the furnace voltage. The goal is to predict the furnace voltage and current based on the history of input and output of the EAF.

\subsection{Experimental process}

In any LS-SVM based modeling, a number of initial decisions are to be made. These include:

- Per-processing of the data: includes choosing the training set and test set. The training data set is selected randomly and the test set is selected continuance after training set.

- The kernel function: the RBF is widely used in different applications for kernel function. And a decision was made to use RBF as a kernel function.

- The parameters: includes all the parameters that used in the LS-SVM algorithm. Some parameters have default values. 
Based on above assumption, the process of the building a least-square-support-vector machine generally is illustrated in Fig (4.1).

There are several advantages and disadvantages associated with using SVM and LS-SVM. The advantages are as follows:

- The prediction accuracy is generally high,

- SVMs provide a good generalization performance,

- Modeling is in high dimensional space by using kernel functions.

- A particular advantage of SVM is the sparseness of the solution ${ }^{[6]}$.

The disadvantages of the SVMs are as follows:

- The training time may be computationally expensive (long training),

- It is difficult to interpret the weights,

- selecting the best kernel function and their parameters is time consuming, 


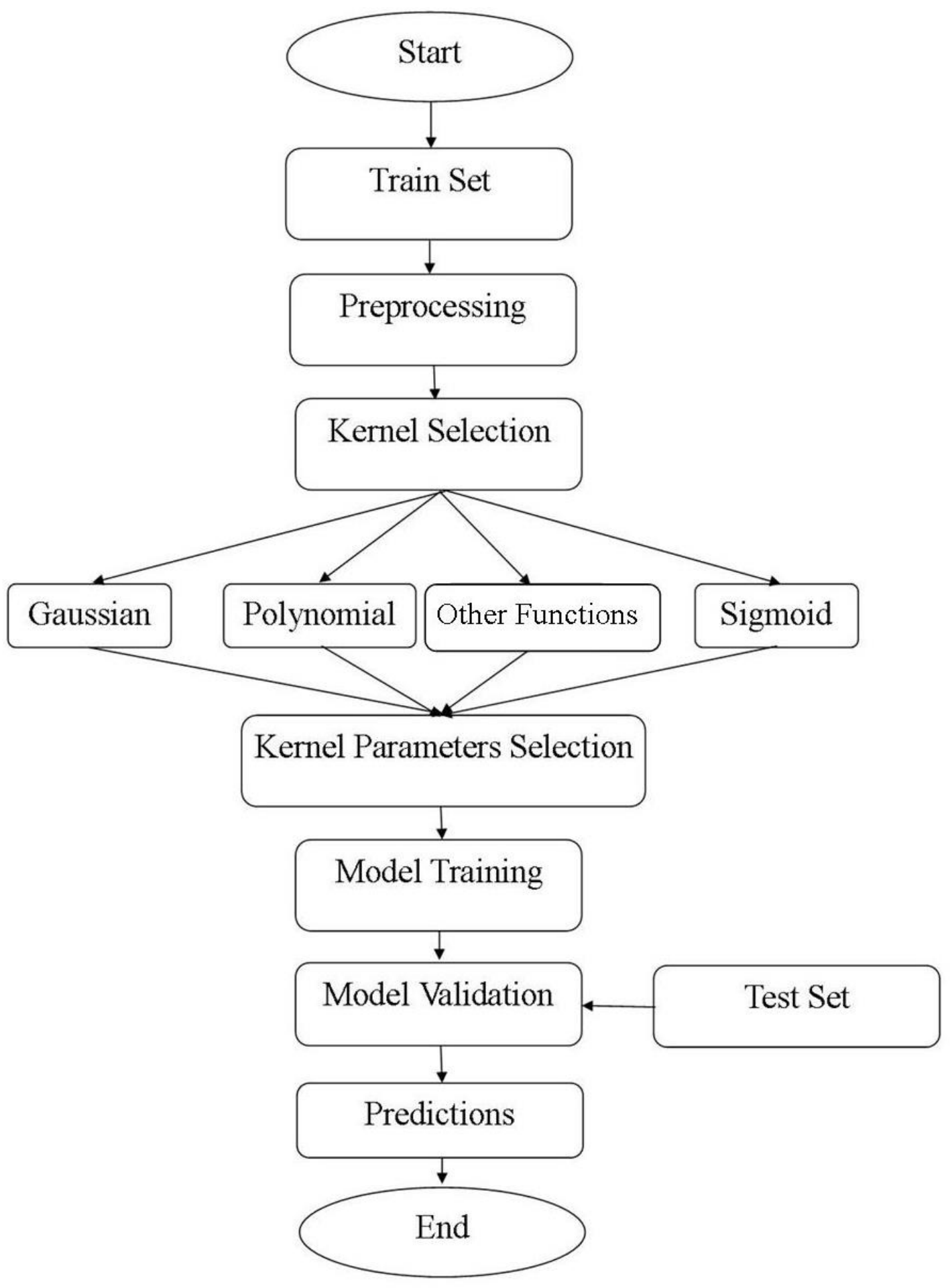

Fig. 4.1. Process of Building the LS-SVM Model 
In this work, the LS-SVM toolbox in MATLAB has been used. The first step in using the LSSVM is to recognize the features of the input and output spaces. Then we need to choose on the structure of the mapping. The purpose of modeling the EAF with LS-SVM is to predict the $\mathrm{V}_{\mathrm{EAF}}$ (the EAF voltage), and $\mathrm{I}_{\mathrm{EAF}}$ (EAF current) as shown below:

$$
\begin{aligned}
V_{E A F}^{t} & =f\left(\left[i^{t}, v^{t-1}\right]\right) \\
I_{E A F}^{t} & =f\left(\left[i^{t-1}, v^{t}\right]\right)
\end{aligned}
$$

Therefore, to model the characteristics of the EAF, we need furnace current and voltage. However, in reality this data may be inadequate and the training process may not converge due to the non-linear $v-i$ characteristic of the arc furnace. Thus, the furnace current derivative, $\partial \mathrm{i}_{\mathrm{EAF}} / \partial \mathrm{t}$, is needed to separate two areas that is illustrated on the $v-i$ characteristics of the electric $\operatorname{arc~(Fig.~1.2).~}$

\subsection{Prediction performance}

Implementation of the LS-SVM for the electric arc furnace includes six steps. First step is the preprocessing. Second step is choosing kernel function and its parameters and after that is training process. Next step after training the data and obtain a model for LS-SVM is a testing

process. Last step is comparing the predicted data with actual data and plotting this comparison. Fig. 4.3 shows the steps involved in building a LS-SVM model for electric arc furnace. 


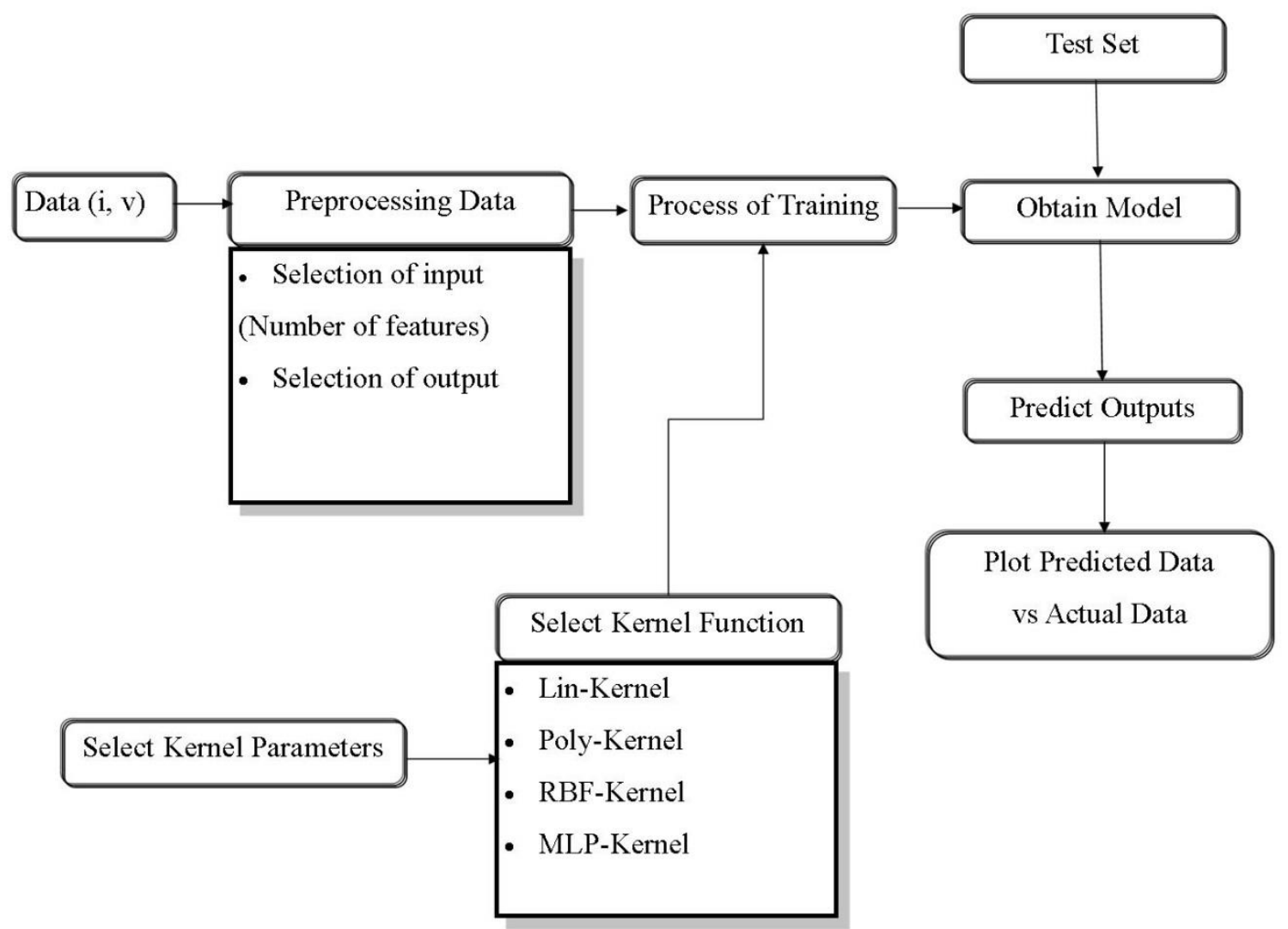

Fig. 4.3. Process of building a LS-SVM model for electric arc furnace in MATLAB

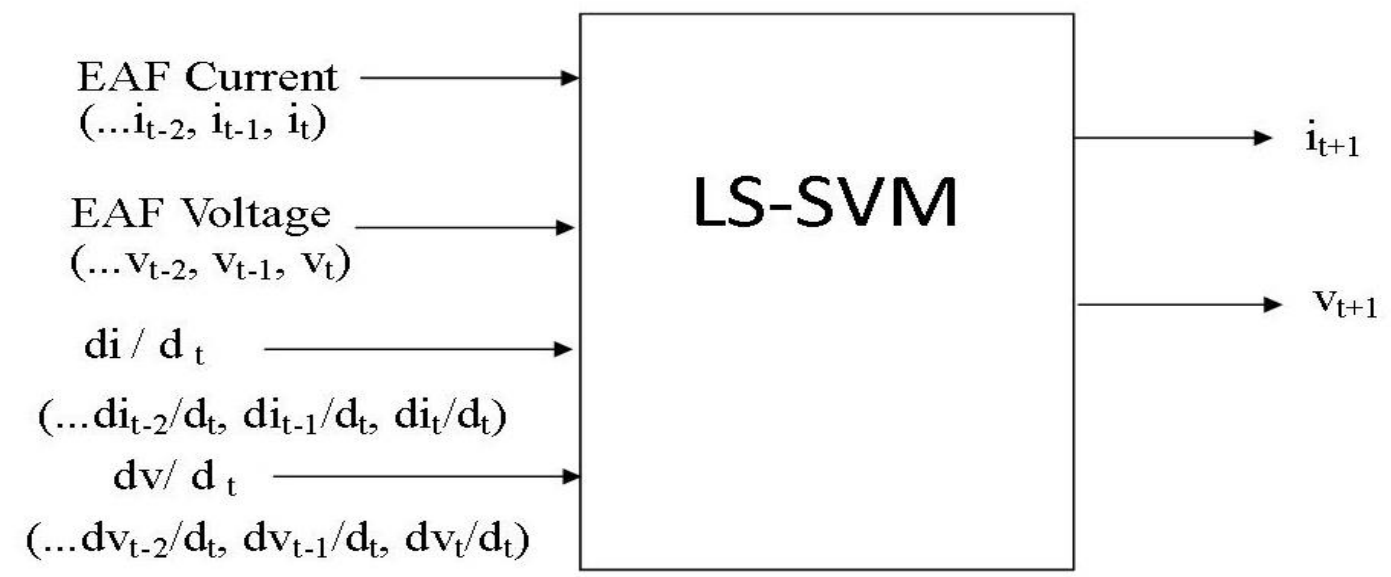

Fig. 4.4. LS-SVM input/output structure 
Fig. 4.4 illustrates the input/output structure used for the LS-SVM modeling for the EAF. We have used the LS-SVM toolbox in MATLAB for this prediction. In this section, most of possible kernel options have been examined. Fig. 4.5 illustrates the 600 pairs of actual data used for the training set, and Fig. 4.6 shows the 600 pairs of actual data used for the test set. We have applied both the RBF and polynomial kernels to predict the voltage and current of the EAF and the results of this experiment have been summarized in Table 4.2. However, in this table, only the best results considering the number of support vector, training accuracy and time have been illustrated. Practically, we could not find a promising method to select the parameters other than trial and error.
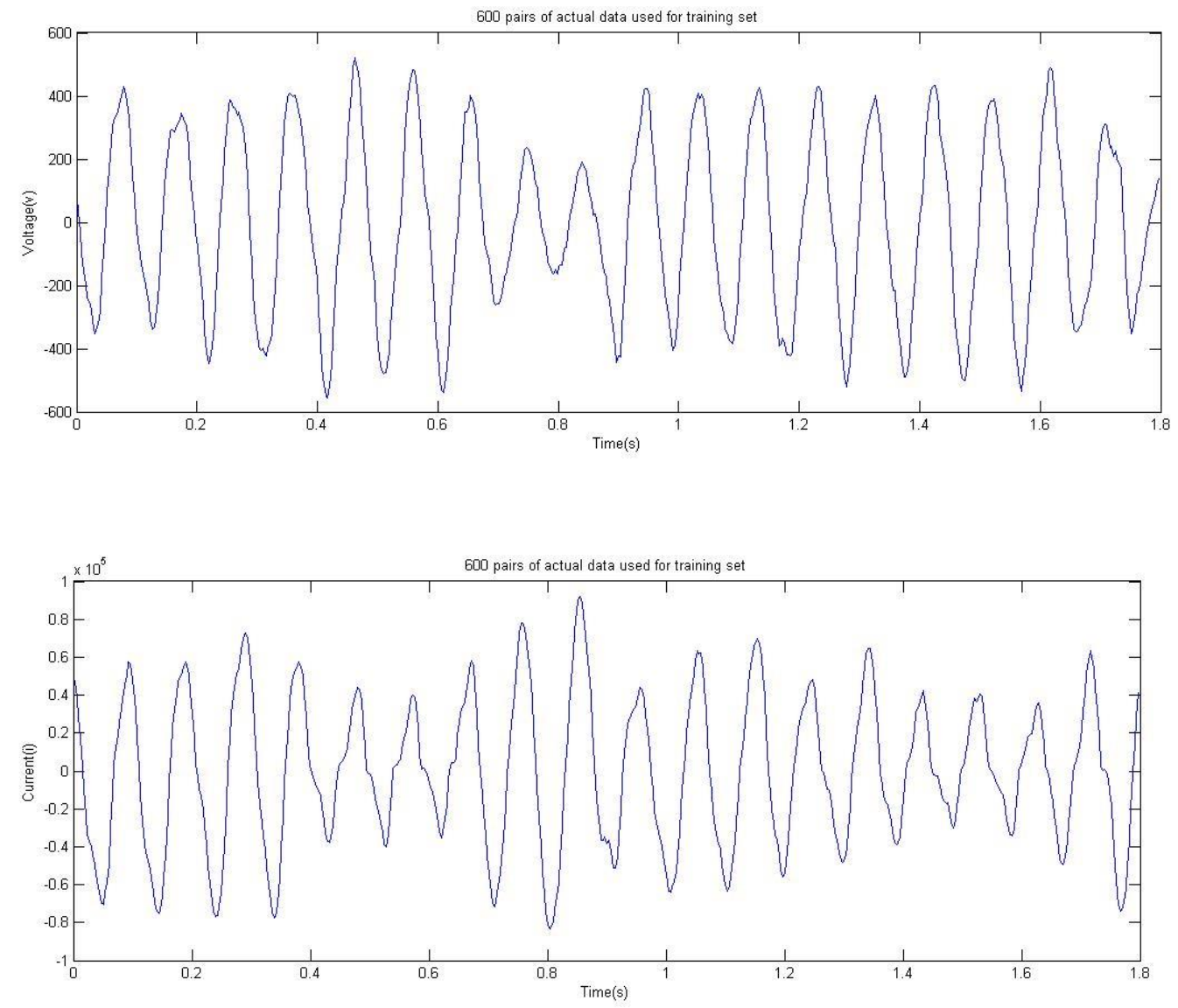

Fig. 4.5. Actual data used as the training set 

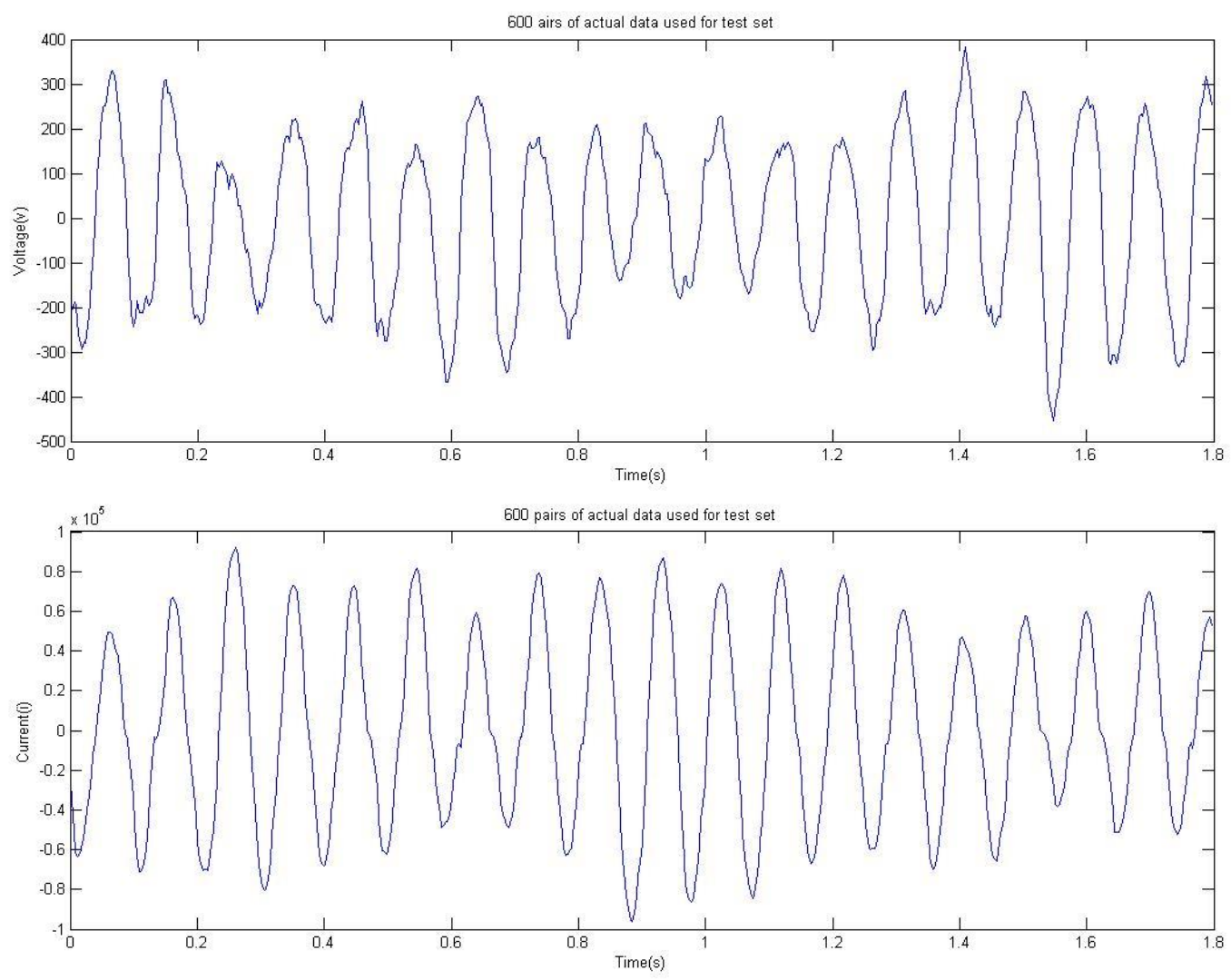

Fig. 4.6. Actual data used as the test set

In the first best attempt in Table 4.2, our input for the training set has 16 features and 1,000 instances. The features for each instance are as follows:

$\left[i_{t-3}, i_{t-2}, i_{t-1}, i_{t}\right.$

$v_{t-3}, v_{t-2}, v_{t-1}, v_{t}$;

$\partial i_{t-3} / \partial t, \partial i_{t-2} / \partial t, \partial \mathrm{i}_{t-1} / \partial t, \partial i_{t} / \partial t$

$\left.\partial v_{t-3} / \partial t, \partial v_{t-2} / \partial t, \partial v_{t-1} / \partial t, \partial v_{t} / \partial t\right]$

The output for training set has 2 features; $\left[i_{t+1} ; v_{t+1}\right]$ 
Fig.4.7 shows the results for prediction of arc current and arc voltage with LS-SVM and compares them with actual data in our test set for the first experiment.
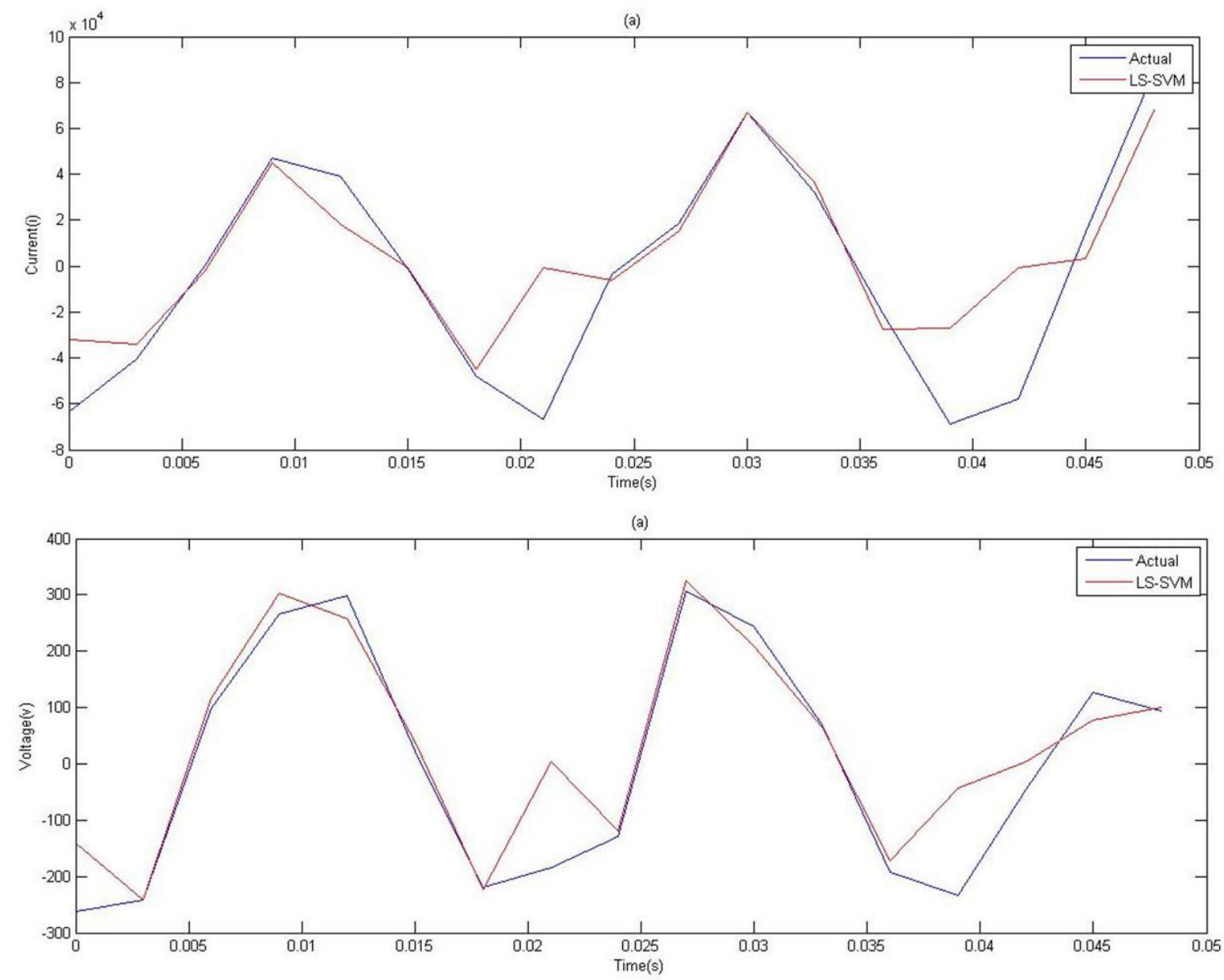

Fig. 4.7. Comparison of the predicted with the actual measurements for arc current and arc voltage:

(a) Dimension of input vector: 16

In the second experiment, which has reliable results, the input for the training set has 12 features and 1,000 instances. The features for each instance are as follows:

$\left[i_{t-3}, i_{t-2}, i_{t-1}, i_{t}\right.$

$v_{t-3}, v_{t-2}, v_{t-1}, v_{t}$

$\left.\partial v_{t-3} / \partial t, \partial v_{t-2} / \partial t, \partial \mathrm{v}_{t-1} / \partial t, \partial v_{t} / \partial t\right]$ 
The output for training set has 2 features; $\left[i_{t+1} ; v_{t+1}\right]$

Fig. 4.8 shows the results for prediction of arc current and arc voltage with LS-SVM and compares them with actual data in our test set for this experiment.
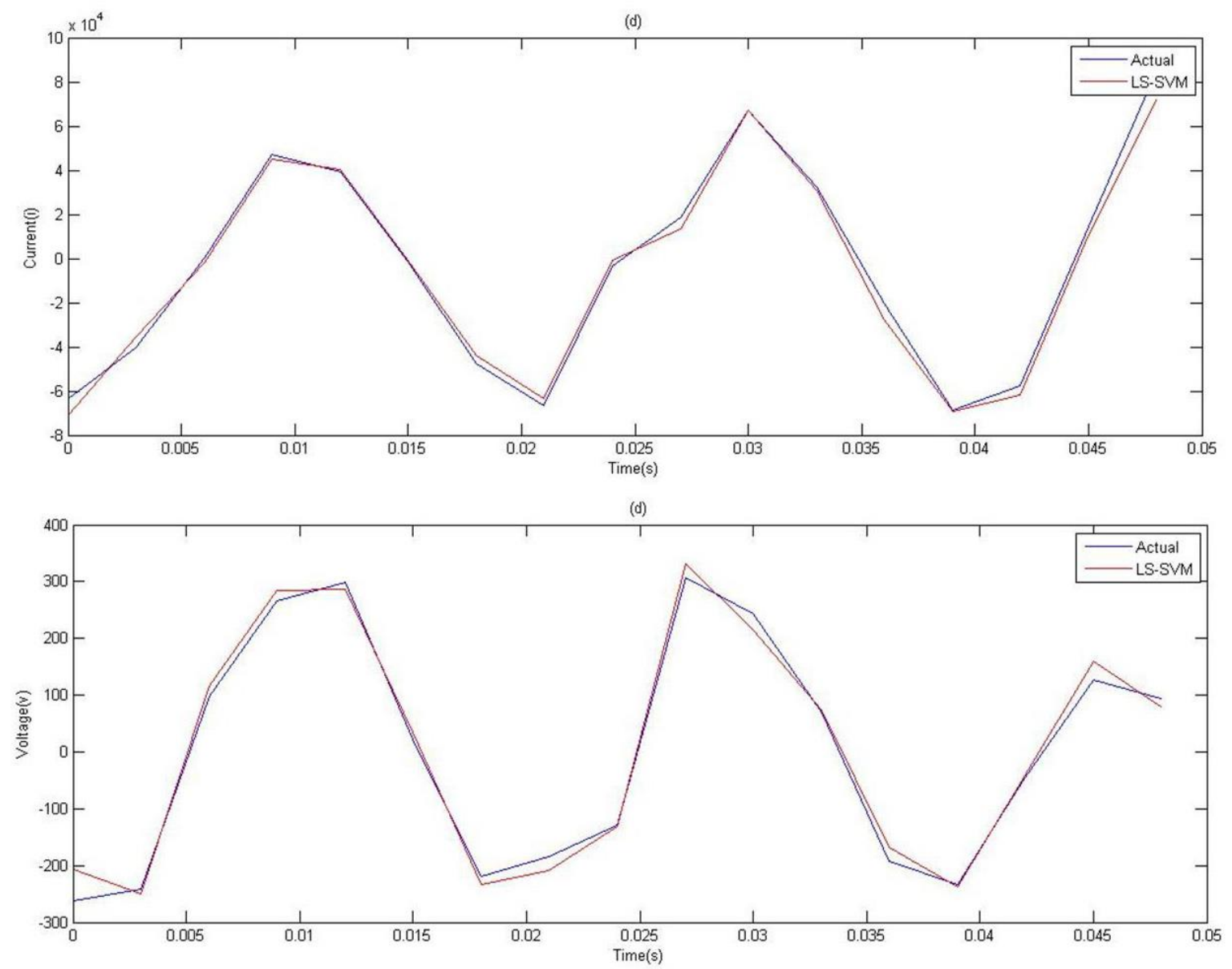

Fig. 4.8. Comparison of the predicted with the actual measurements for arc current and arc voltage:

(b) Dimension of input vector: 12

In the third experiment, our input for the training set has 12 features and 1,000 instances. The features for each instance are as follows: 
$\left[i_{t-3}, i_{t-2}, i_{t-1}, i_{t}\right.$

$v_{t-3}, v_{t-2}, v_{t-1}, v_{t}$

$\left.\partial i_{t-3} / \partial t, \partial i_{t-2} / \partial t, \partial i_{t-1} / \partial t, \partial i_{t} / \partial t\right]$

The output for training set has 2 features; $\left[i_{t+1} ; v_{t+1}\right]$

Fig. 4.9 illustrates the results for prediction of arc current and arc voltage with LS-SVM and compares them with actual data in our test set for third experiment in table 4.2.
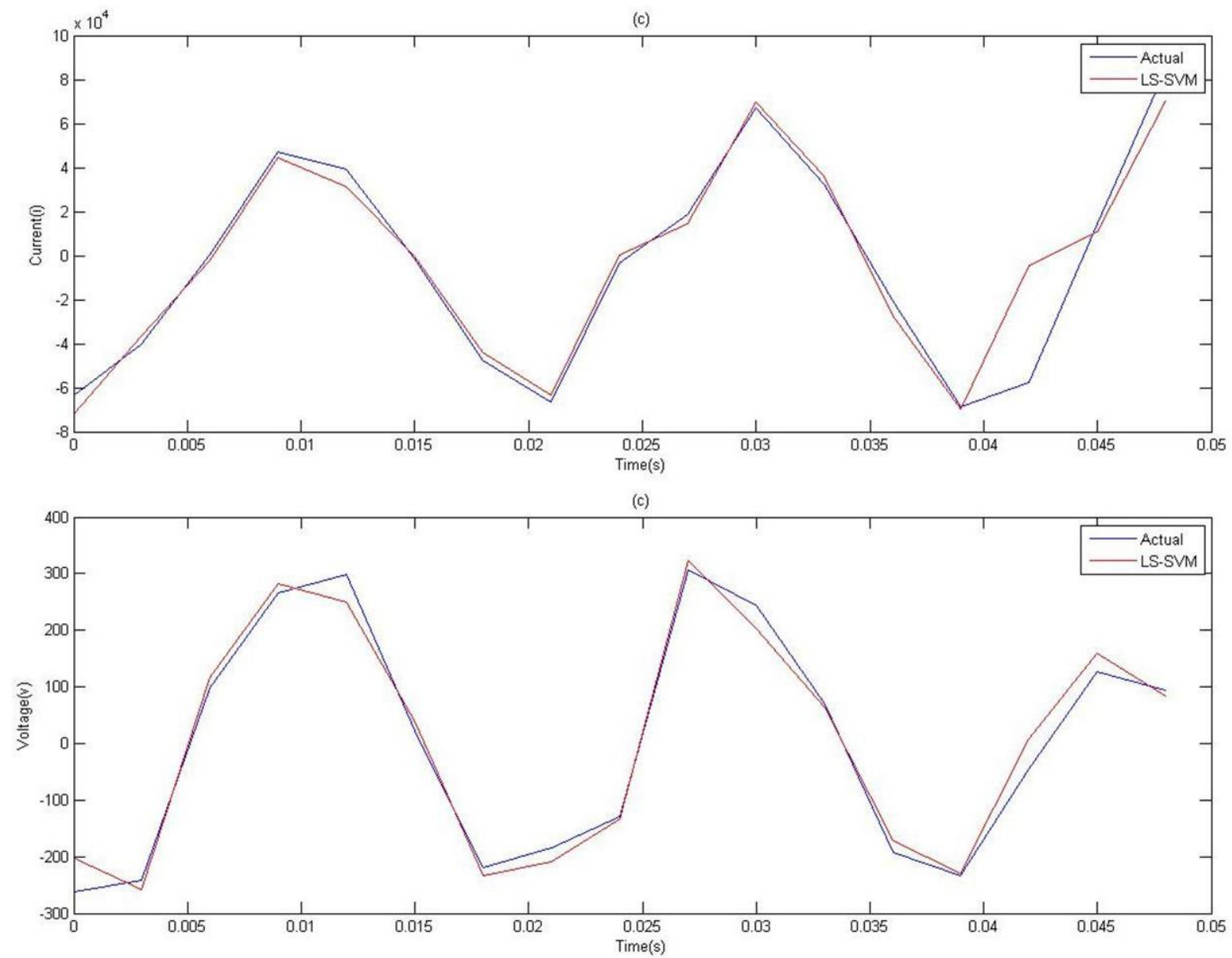

Fig. 4.9. Comparison of the predicted with the actual measurements for arc current and arc voltage:

(c) Dimension of input vector: 12 
In the last experiment in Table 4.2, our input for the training set has 8 features and 1,000 instances. The features for each instance are as follows:

$\left[i_{t-3,}, i_{t-2}, i_{t-1}, i_{t}\right.$

$$
\left.v_{t-3}, v_{t-2}, v_{t-1}, v_{\mathrm{t}}\right]
$$

The output for training set has 2 features; $\left[i_{t+1}, v_{t+1}\right]$

Fig. 4.10 displays the results for prediction of arc current and arc voltage with LS-SVM and compares them with actual data in our test set for the last experiment in this section.
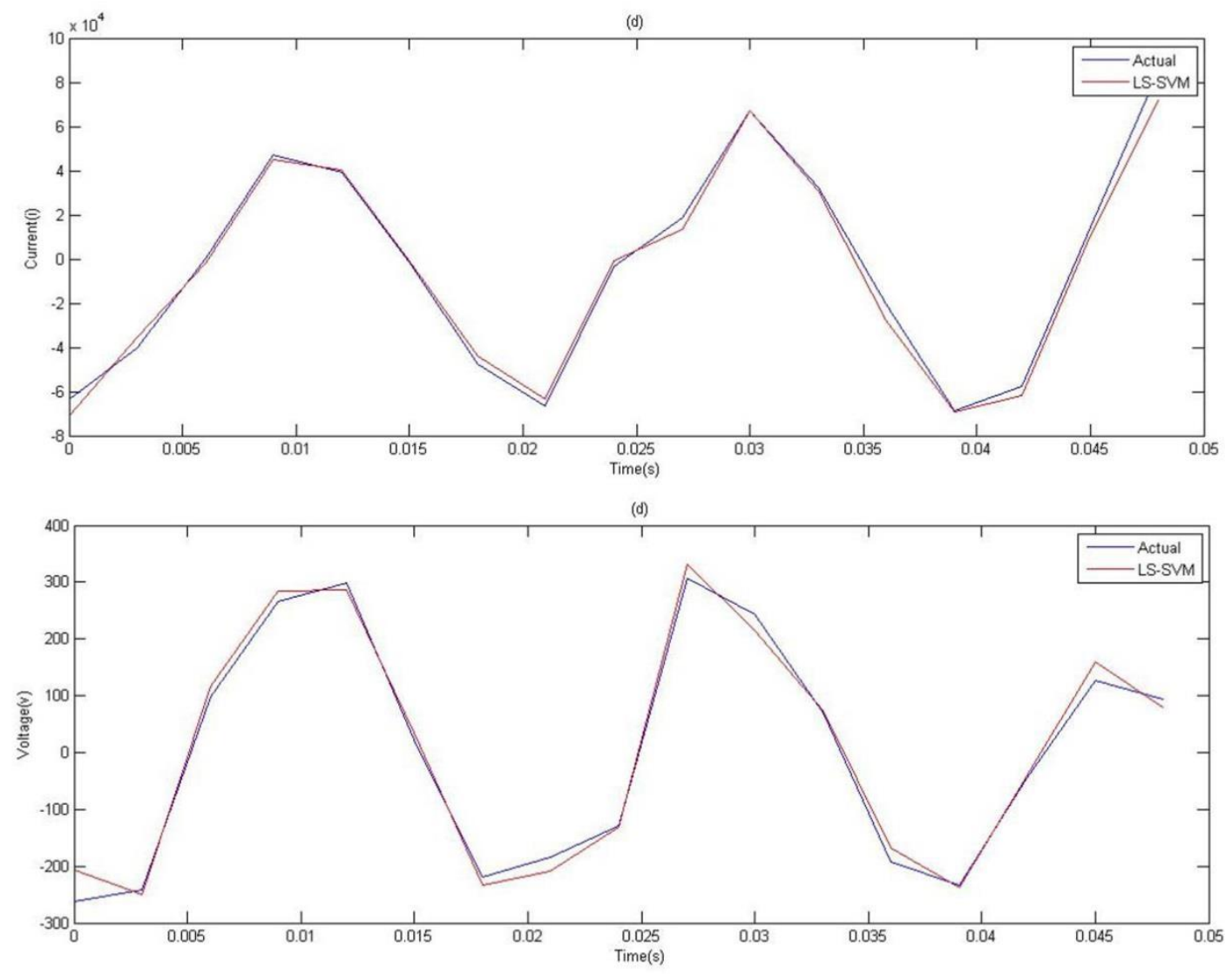

Fig. 4.10. Comparison of the predicted with the actual measurements for arc current and arc voltage:

(d) Dimension of input vector: 8 
The best kernel function in all of the experiments is RBF-kernel. The best sigma is 0.4 and the best gamma is 10 .

For the comparison we have used the mean absolute percentage error or deviation (MAPE/MAPD) and non-dimensional index error (NDEI). MAPE is the absolute value of the average of all of the percentage errors. MAPE or MAPD is defined by the following formula:

$M A P E=\frac{1}{N} \sum_{i=1}^{N} \frac{\left(\left|A_{t}-P_{t}\right|\right)}{\left|A_{t}\right|}$

where $A t$ and $P t$ are the actual value and the predicted value, respectively.

NDEI is defined as the root mean square error of modeling normalized divided by the standard deviation of the target data [1] and it can be presented by the following formula:

$$
N D E I=\sqrt{\frac{1}{N} \sum_{i=1}^{N}\left(A_{t}-P_{t}\right)^{2}} / \sigma\left(A_{t}\right)
$$

Table 4.1. Comparison between prediction the $v-i$ with different dimension of Inputs-outputs for RBF kernels

\begin{tabular}{|c|c|c|c|c|c|c|c|}
\hline $\begin{array}{c}\text { Number } \\
\text { of } \\
\text { training } \\
\text { data }\end{array}$ & $\begin{array}{c}\text { Dimension } \\
\text { input } \\
\text { vector }\end{array}$ & $\begin{array}{c}\text { Dimension } \\
\text { output } \\
\text { vector }\end{array}$ & $\begin{array}{c}\text { Number } \\
\text { of test } \\
\text { data }\end{array}$ & $\begin{array}{c}\text { NDEI } \\
\text { current } \\
\left(i_{t+1}\right)\end{array}$ & $\begin{array}{c}\text { NDEI } \\
\text { voltage } \\
\left(v_{t+1}\right)\end{array}$ & $\begin{array}{c}\text { MAPE } \\
\text { current } \\
\left(i_{t+1}\right)\end{array}$ & $\begin{array}{c}\text { MAPE } \\
\text { voltage } \\
\left(v_{t+1}\right)\end{array}$ \\
\hline 1000 & 16 & 2 & 1000 & 0.65989 & 0.612953 & 0.0014288 & 0.131706 \\
\hline 1000 & 12 & 2 & 1000 & 0.332551 & 0.227595 & 0.0109027 & 0.001244 \\
\hline 1000 & 12 & 2 & 1000 & 0.183048 & 0.271530 & 0.0038800 & 0.004859 \\
\hline 1000 & 8 & 2 & 1000 & 0.086395 & 0.095013 & 0.0024180 & 0.000366 \\
\hline
\end{tabular}


Based on the Table 4.2, the best result to predict both arc voltage and arc current is the model that has the training data with 8 features and test data with 2 features. Thus, we decided to build another structure for LS-SVM, which has the past history of current and voltage as the inputs, and the predicted future current and voltage as the outputs (Fig. 4.11), but each time we have different size of window for the input and we have been compared all of the results to obtain the best result for prediction both $i_{t+1}$ and $v_{t+1}$.

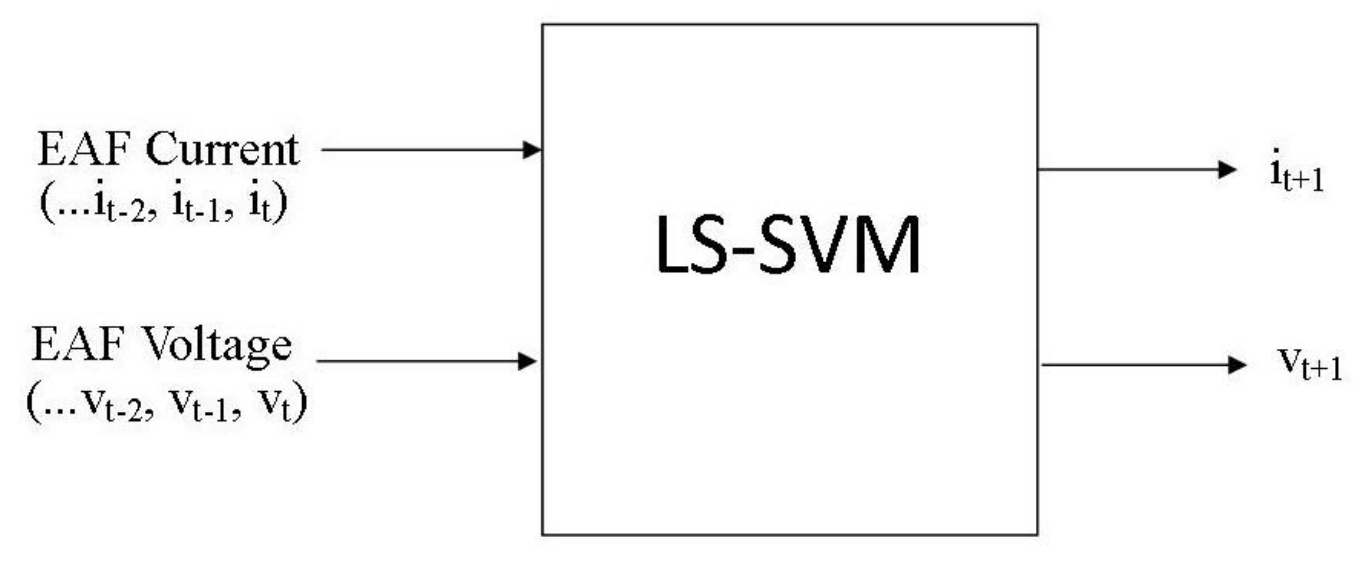

Fig. 4.11. New LS-SVM input/output structure

In the following experiment, we provide four different models -- with different inputs but the same output -- to predict the arc current and voltage. In the first attempt, the input for the training data is $\left[i_{t} ; v_{t}\right]$ and the output is $\left[i_{t+1} ; v_{t+1}\right]$. For the second experiment, $\left[i_{t-1}, i_{t} ; v_{t-1}, v_{t}\right]$ and $\left[i_{t+1} ; v_{t+1}\right]$ have been used as the input/output. The input for the third model is $\left[i_{t-2}, i_{t-1}, i_{t} ; v_{t-2}, v_{t-1}, v_{t}\right]$. In the last experiment, the input for training data is $\left[i_{t-3}, i_{t-2}, i_{t-1}, i_{t} ; v_{t-3}, v_{t-2}, v_{t-1}, v_{t}\right]$.

The results for these experiments are summarized in Table 4.3. Fig. 4.12 depicts the comparison between predicted and actual values to predict arc current for all four results in the Table 4.3. 
Fig. 4.13 illustrates the comparison between predicted and actual values to predict arc voltage for all four results that are discussed in Table 4.3.

Table 4.2. Comparison the results with different inputs

\begin{tabular}{|c|c|c|c|c|c|c|}
\hline Input & $\begin{array}{c}\text { Dimension } \\
\text { of input } \\
\text { vector }\end{array}$ & $\begin{array}{c}\text { Dimension } \\
\text { of output } \\
\text { vector }\end{array}$ & $\begin{array}{c}\text { NDEI } \\
\text { current } \\
i_{t+1}\end{array}$ & $\begin{array}{c}\text { NDEI } \\
\text { voltage } \\
v_{t+1}\end{array}$ & $\begin{array}{c}\text { MAPE } \\
\text { current } \\
i_{t+1}\end{array}$ & $\begin{array}{c}\text { MAPE } \\
\text { voltage } \\
v_{t+1}\end{array}$ \\
\hline$\left[i_{t}, v_{t}\right]$ & 2 & 2 & 0.231427 & 0.229131 & 0.001362 & 0.000556 \\
\hline$\left[i_{t-1}, i_{t} ; v_{t-1}, v_{t}\right]$ & 4 & 2 & 0.063267 & 0.079526 & 0.001191 & 0.001247 \\
\hline$\left[i_{t-2}, i_{t-1}, i_{t} ; v_{t-2}, v_{t-1}, v_{t}\right]$ & 6 & 2 & 0.053655 & 0.074696 & 0.000354 & 0.001462 \\
\hline$\left[i_{t-3,}, i_{t-2}, i_{t-1}, i_{t} ; v_{t-3}, v_{t-2}, v_{t-1}, v_{t}\right]$ & 8 & 2 & 0.077482 & 0.094348 & 0.003262 & 0.001339 \\
\hline
\end{tabular}



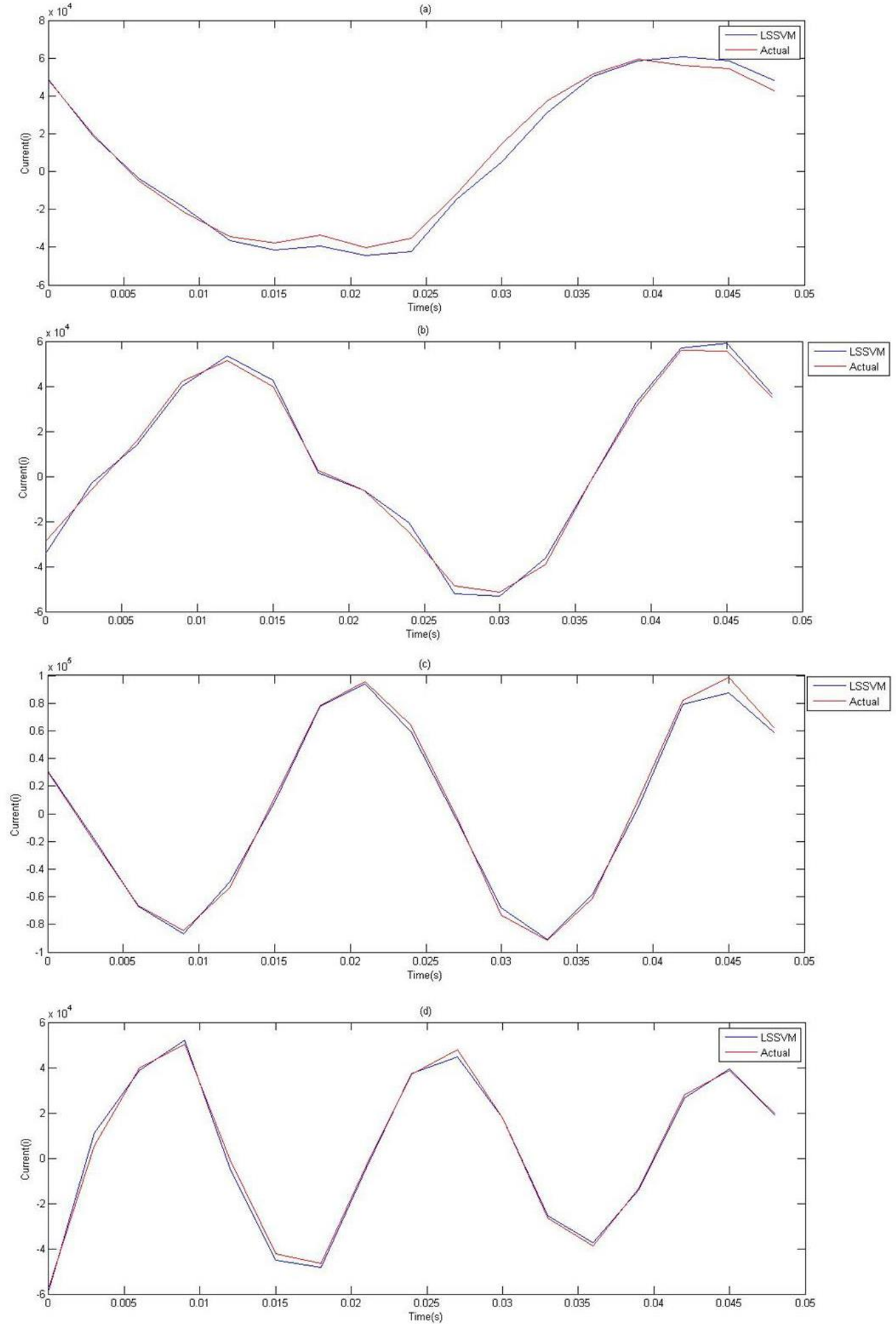

Fig. 4.12. Comparison of the predicted vs actual measurements for arc current where the input is:

(a) $\left[i_{t}, v_{t}\right]$, (b) $\left[i_{t-1}, i_{t} ; v_{t-1}, v_{t}\right]$

(c) $\left[i_{t-2}, i_{t-1}, i_{t} ; v_{t-2}, v_{t-1}, v_{t}\right]$, (d) $\left[i_{t-3}, i_{t-2}, i_{t-1}, i_{t} ; v_{t-3}, v_{t-2}, v_{t-1}, v_{t}\right]$ 

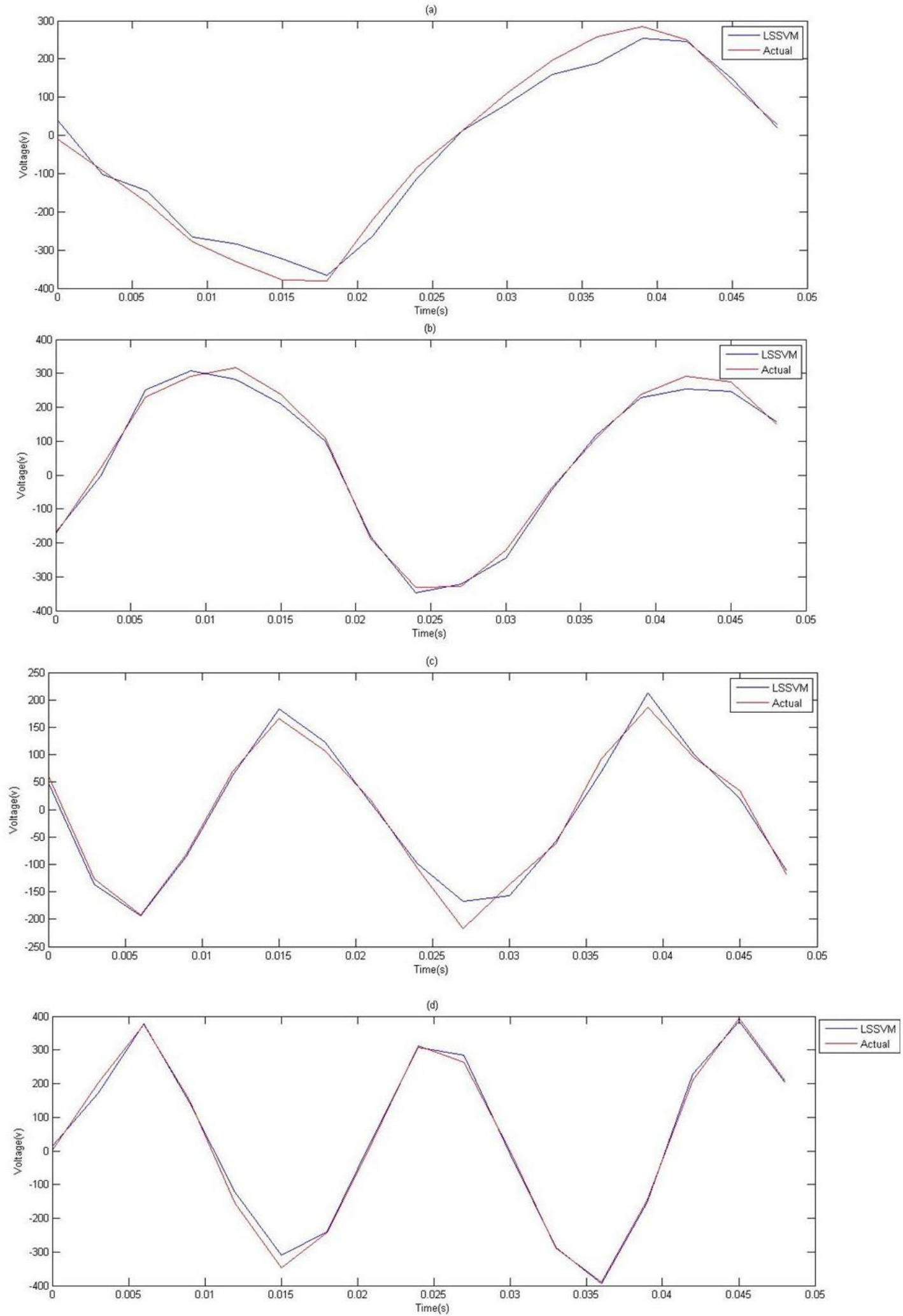

Fig. 4.13. Comparison of the predicted and actual values for arc voltage where input is (a) $\left[i_{t}, v_{t}\right]$, (b) $\left[i_{t-1}, i_{t} ; v_{t-1}, v_{t}\right]$

(c) $\left[i_{t-2}, i_{t-1}, i_{t} ; v_{t-2}, v_{t-1}, v_{t}\right]$, (d) $\left[i_{t-3}, i_{t-2}, i_{t-1}, i_{t} ; v_{t-3}, v_{t-2}, v_{t-1}, v_{t}\right]$ 
To compare the predicted values with the actual values in our different model implementations for predicting the arc current, we have compared 300 data pairs for predicted and actual values for the current of the EAF in a scatter plot. The solid line represents the best fit line obtained from linear regression with the corresponding $95 \%$ confidence interval is depicted with the broken line. Fig. 4.14 displays this linear regression between the predicted and actual data for the model that has the $\left[i_{t} ; v_{t}\right]$ as an input for training. The second diagram in Fig. 4.15, we have the model that has $\left[i_{t-1}, i_{t} ; v_{t-1}, v_{t}\right]$ as an input vector for training set. The third one, Fig. 4.16 illustrates the linear regression between the predicted and actual data for the LS-SVM model that has $\left[i_{t-2}, i_{t-1}, i_{t} ; v_{t-2}, v_{t-1}, v_{t}\right]$ as an input to predict the arc current and the last one, Fig. 4.17 shows the linear regression for the model with $\left[i_{t-3}, i_{t-2}, i_{t-1}, i_{t} ; v_{t-3}, v_{t-2}, v_{t-1}, v_{t}\right]$ as an input vector to predict the arc current and also this model has the best results. 


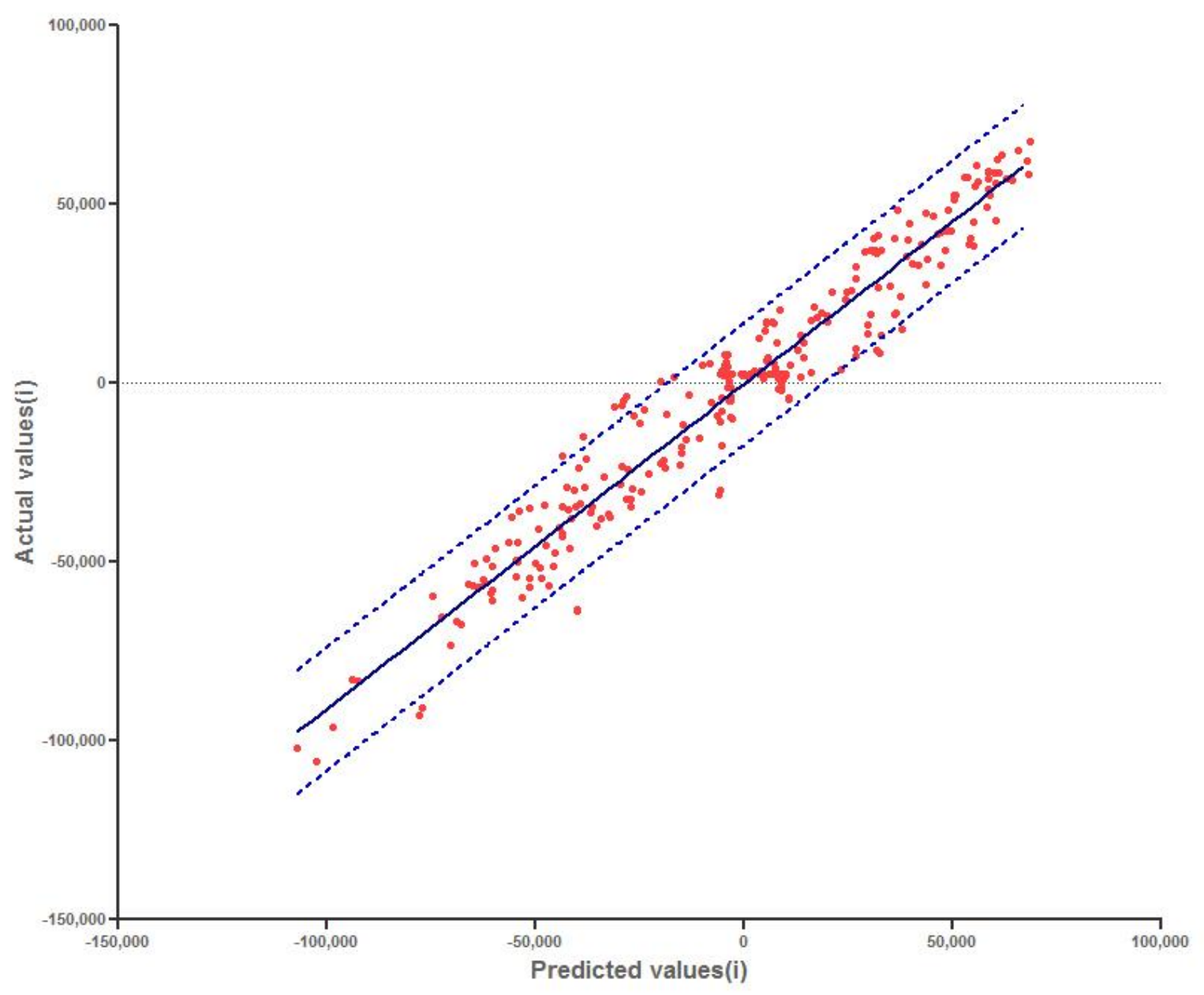

Fig. 4.14. Linear regression with the corresponding 95\% confidence interval between predicted and actual data for arc current using $\left[i_{t} ; v_{t}\right]$ as an input vector 


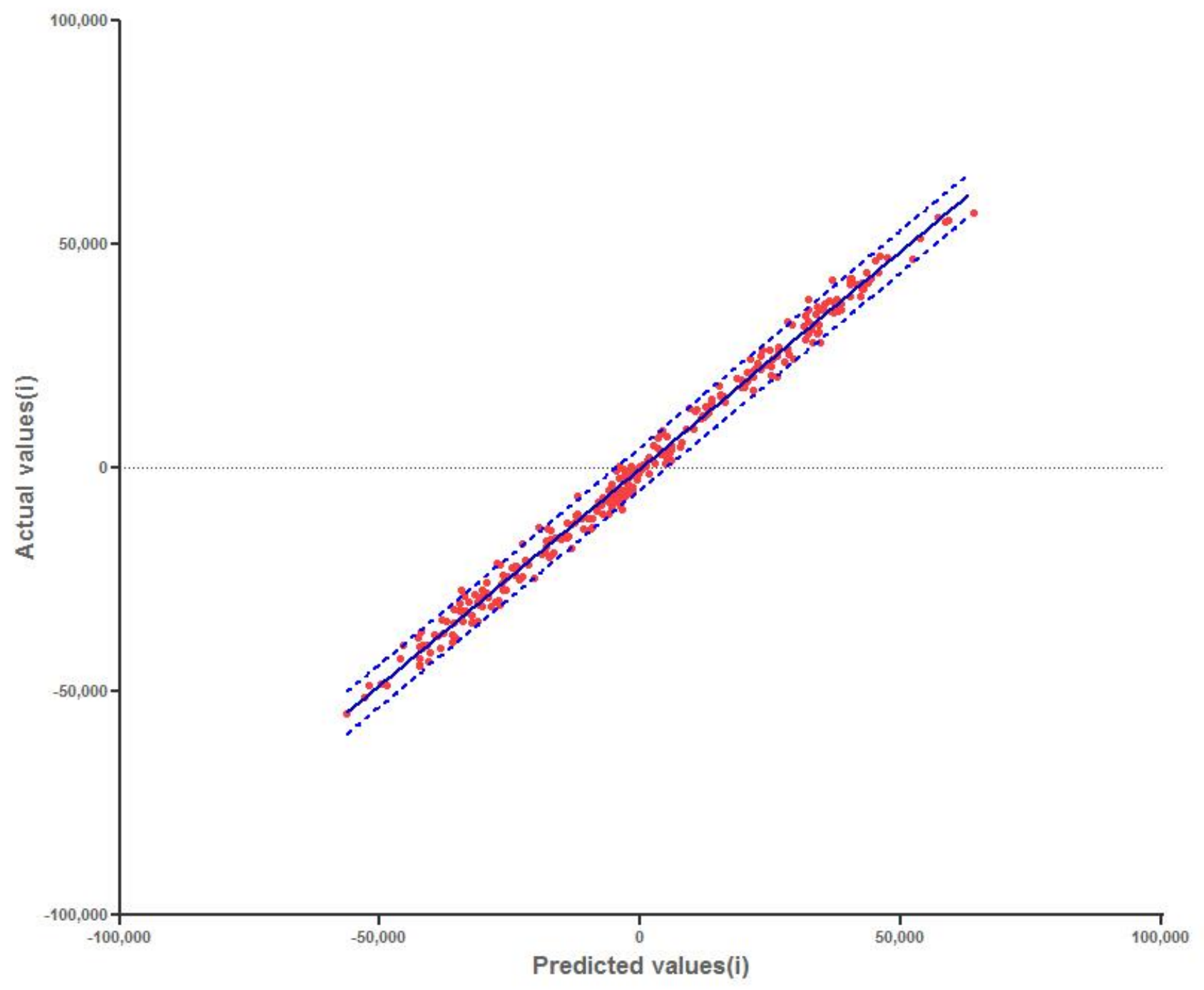

Fig. 4.15. Linear regression with the corresponding 95\% confidence interval between predicted and actual data for arc current using $\left[i_{t-1}, i_{t} ; v_{t-1}, v_{t}\right]$ as an input vector 


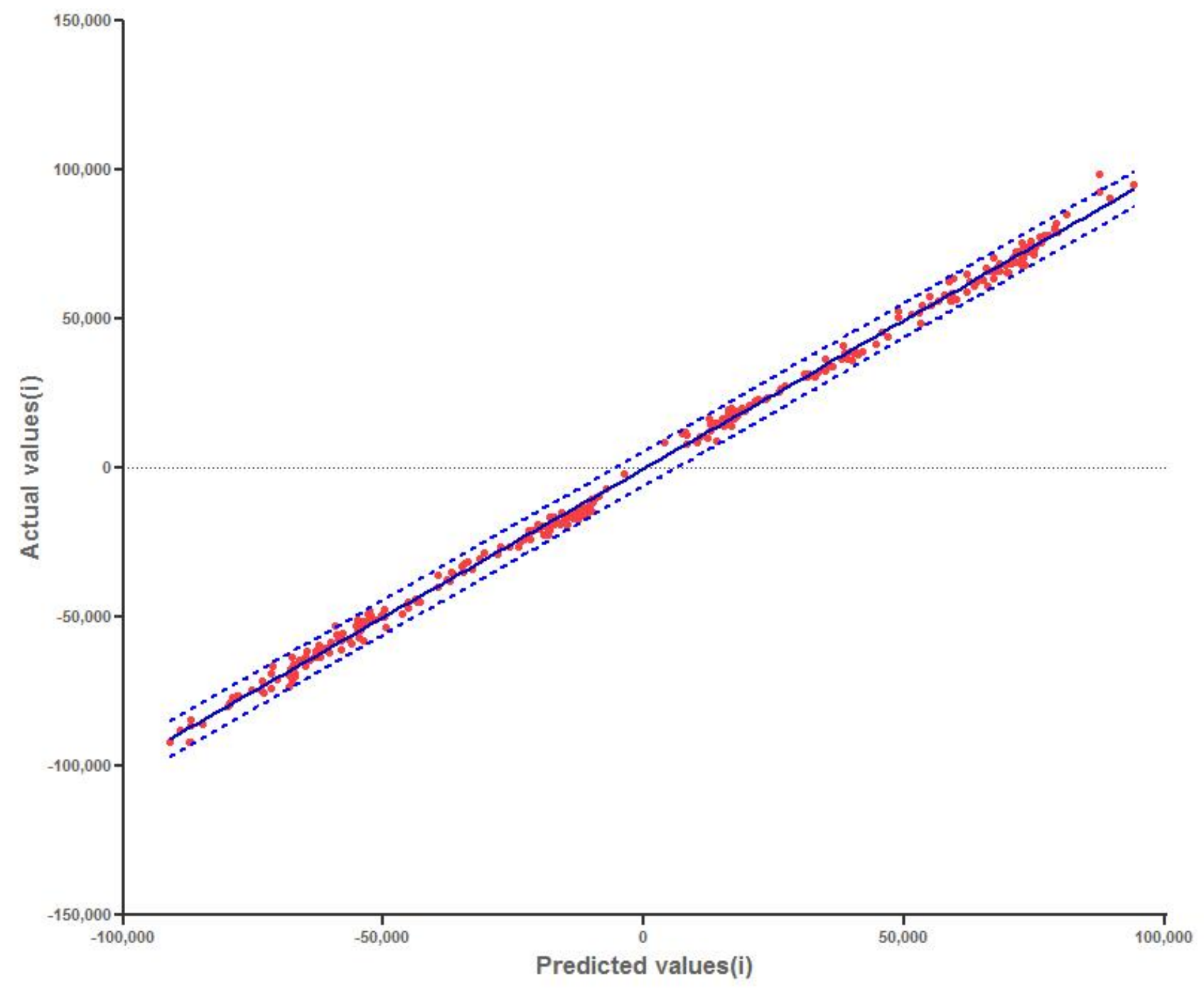

Fig. 4.16. Linear regression with the corresponding 95\% confidence interval between predicted and actual data for arc current using $\left[i_{t-2}, i_{t-1}, i_{t} ; v_{t-2}, v_{t-1}, v_{t}\right]$ as an input vector 


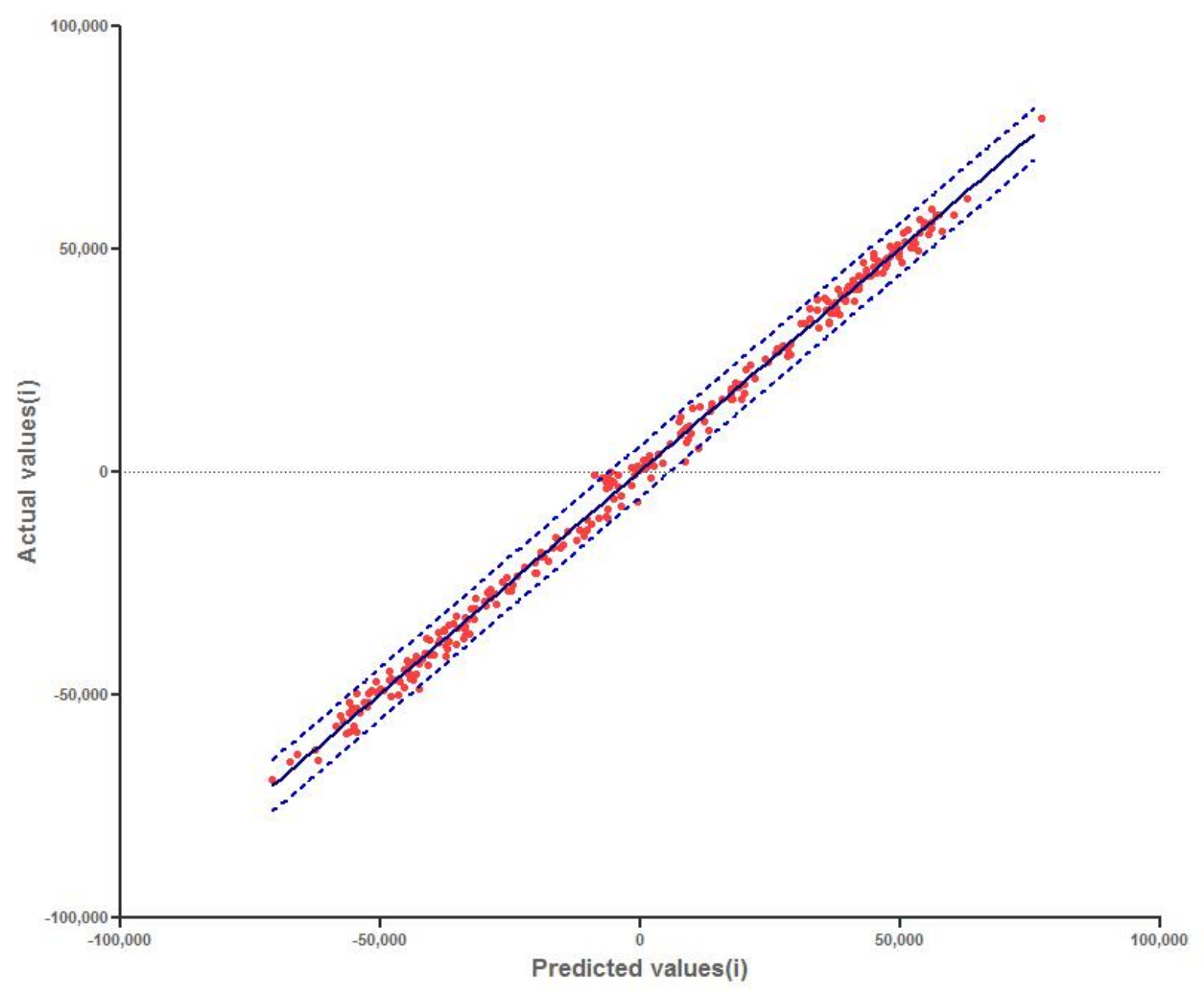

Fig. 4.17. Linear regression with the corresponding 95\% confidence interval between predicted and actual data for arc current using $\left[i_{t-3}, i_{t-2}, i_{t-1}, i_{t} ; v_{t-3}, v_{t-2}, v_{t-1}, v_{t}\right]$ as an input vector

Table 4.4 illustrates the comparison the slope of best fit values and slope of $95 \%$ confidence intervals between two best models (Fig. 4.16, and Fig. 4.17) that used LS-SVM to predict the arc current. Since the slope for both the best fit values and 95\% interval slopes are tighter in the model that used $\left[i_{t-3}, i_{t-2}, i_{t-1}, i_{t} ; v_{t-3}, v_{t-2}, v_{t-1}, v_{t}\right]$ as an input vector for training set, it is our best LSSVM model to predict the arc current. 
Table 4.3. Comparison of the slope of the best fit line and $95 \%$ confidence intervals between two best models to predict the arc current

\begin{tabular}{|c|c|c|}
\hline Input & $\begin{array}{c}\text { Slope } \\
\text { Best-fit values }\end{array}$ & $\begin{array}{c}\text { Slope } \\
\text { 95\% Confidence Intervals }\end{array}$ \\
\hline$\left[i_{t-2}, i_{t-1}, i_{t} ; v_{t-2}, v_{t-1}, v_{t}\right]$ & $0.9975 \pm 0.002555$ & 0.9925 to 1.003 \\
\hline$\left[i_{t-3,3} i_{t-2}, i_{t-1,}, i_{t} ; v_{t-3,}, v_{t-2}, v_{t-1}, v_{t}\right]$ & $0.9986 \pm 0.003461$ & 0.9918 to 1.005 \\
\hline
\end{tabular}

To compare the results between different models to predict the furnace voltage, we have compared 300 data pairs for predicted and actual values for the voltage of the EAF in a scatter plot. The solid line represents the best fit line obtained from linear regression with the corresponding 95\% confidence interval is depicted with the broken line. Fig. 4.18 displays this linear regression between the predicted and actual data for the model that has the $\left[i_{t} ; v_{t}\right]$ as an input for training. The second diagram in Fig. 4.19, we have the model that has $\left[i_{t-1}, i_{t} ; v_{t-1}, v_{t}\right]$ as an input vector for training set. The third one, Fig. 4.20 illustrates the linear regression between the predicted and actual data for the LS-SVM model that has $\left[i_{t-2}, i_{t-1}, i_{t} ; v_{t-2}, v_{t-1}, v_{t}\right]$ as an input to predict the arc voltage and the last one, Fig. 4.21 shows the linear regression for the model with $\left[i_{t-3}, i_{t-2}, i_{t-1}, i_{t} ; v_{t-3}, v_{t-2}, v_{t-1}, v_{t}\right]$ as an input vector to predict the arc voltage and also this model has the best results. 


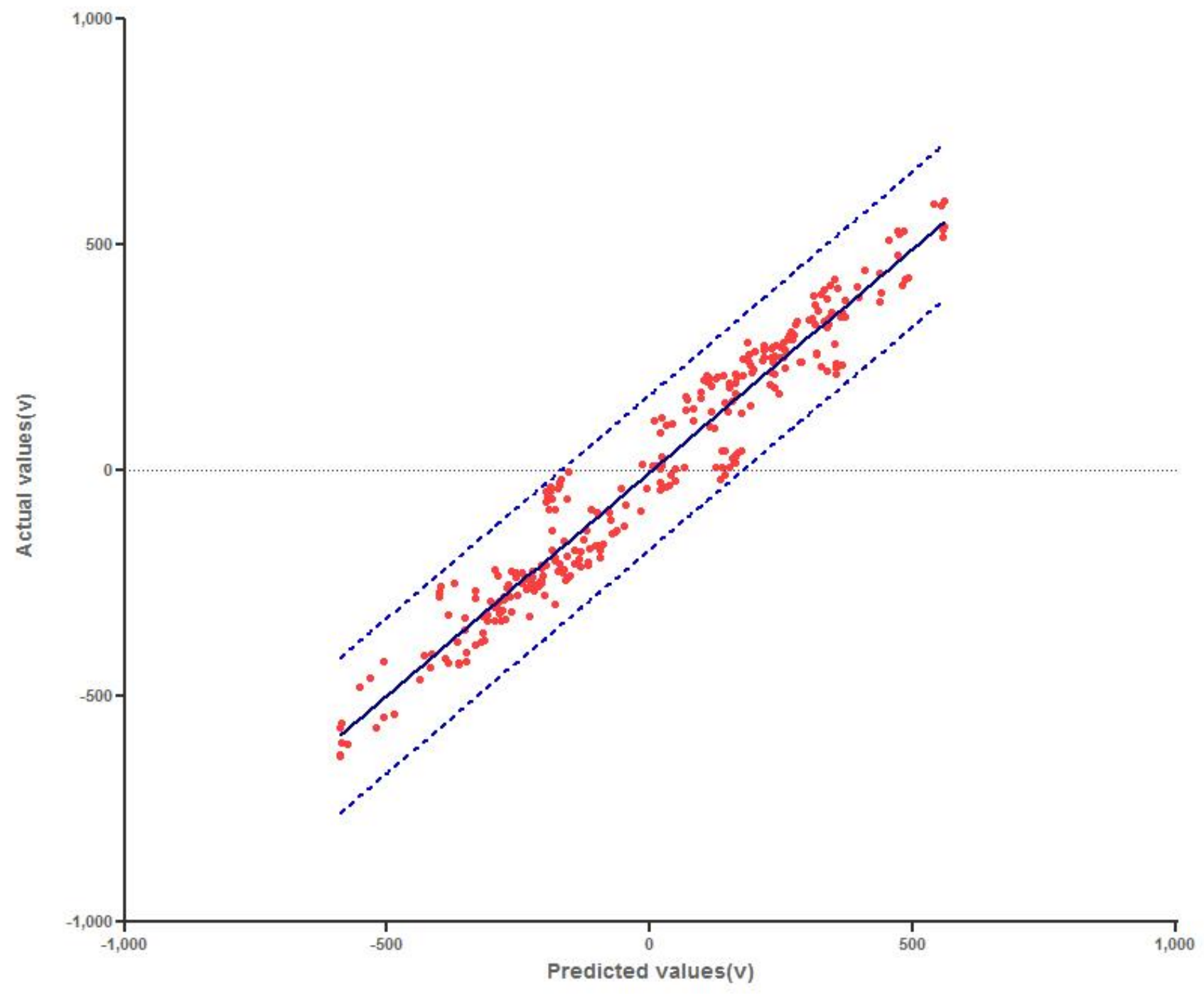

Fig. 4.18. Linear regression with the corresponding 95\% confidence interval between predicted and actual data for arc voltage using $\left[i_{t} ; v_{t}\right]$ as an input vector 


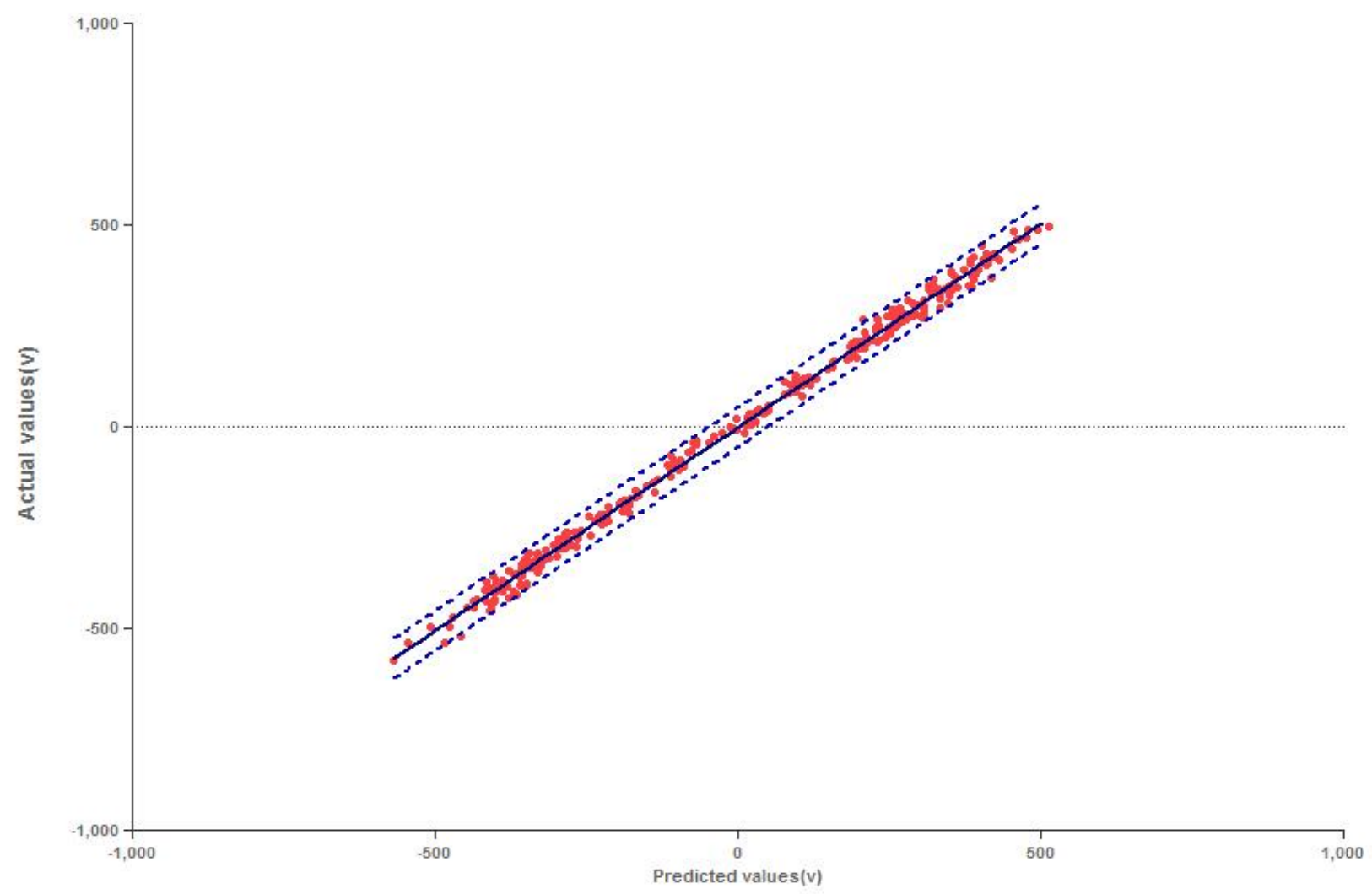

Fig. 4.19. Linear regression with the corresponding $95 \%$ confidence interval between predicted and actual data for arc voltage using $\left[i_{t-1}, i_{t} ; v_{t-1}, v_{t}\right]$ as an input vector 


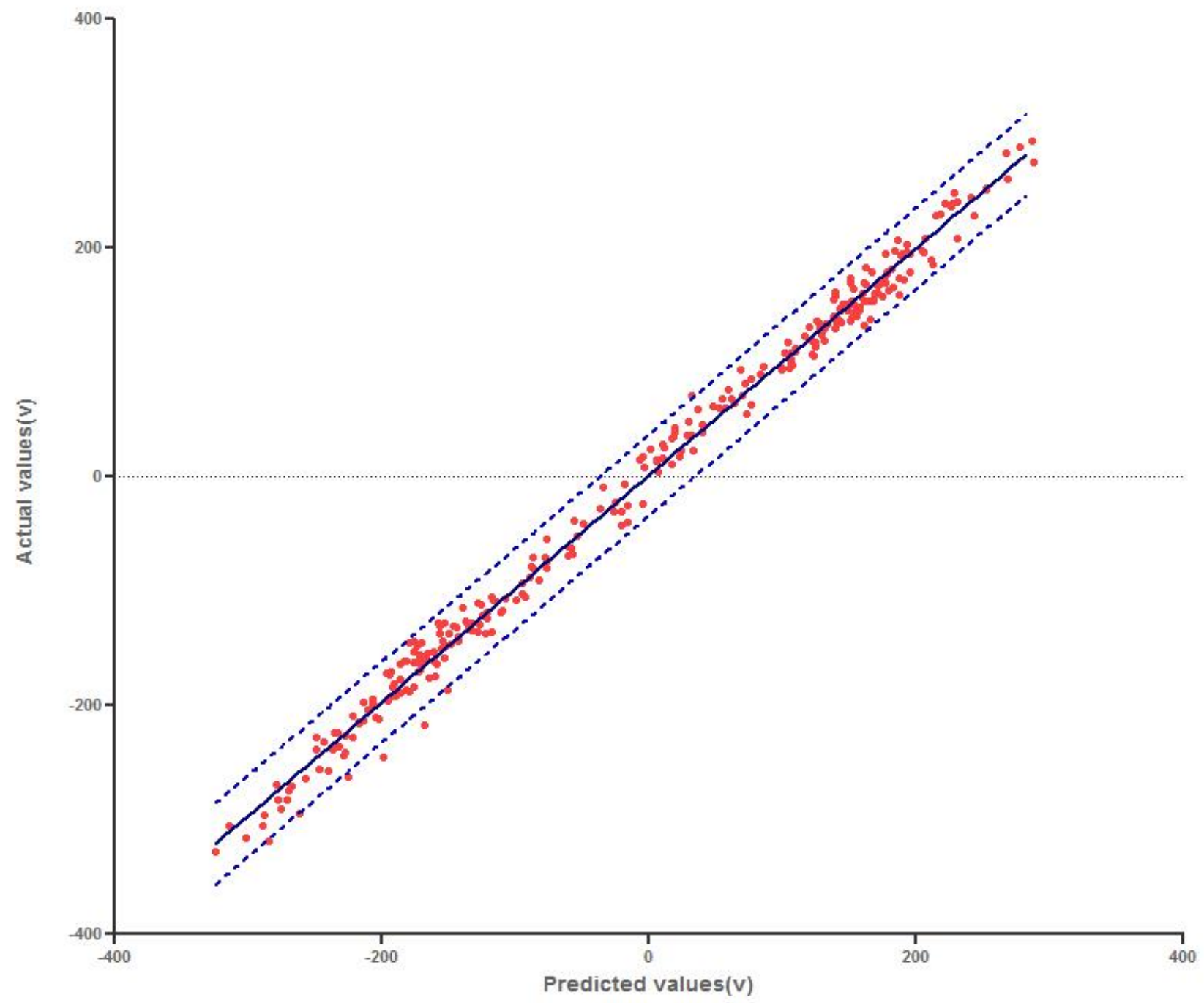

Fig. 4.20. Linear regression with the corresponding 95\% confidence interval between predicted and actual data for arc voltage using $\left[i_{t-2}, i_{t-1}, i_{t} ; v_{t-2}, v_{t-1}, v_{t}\right]$ as an input vector 


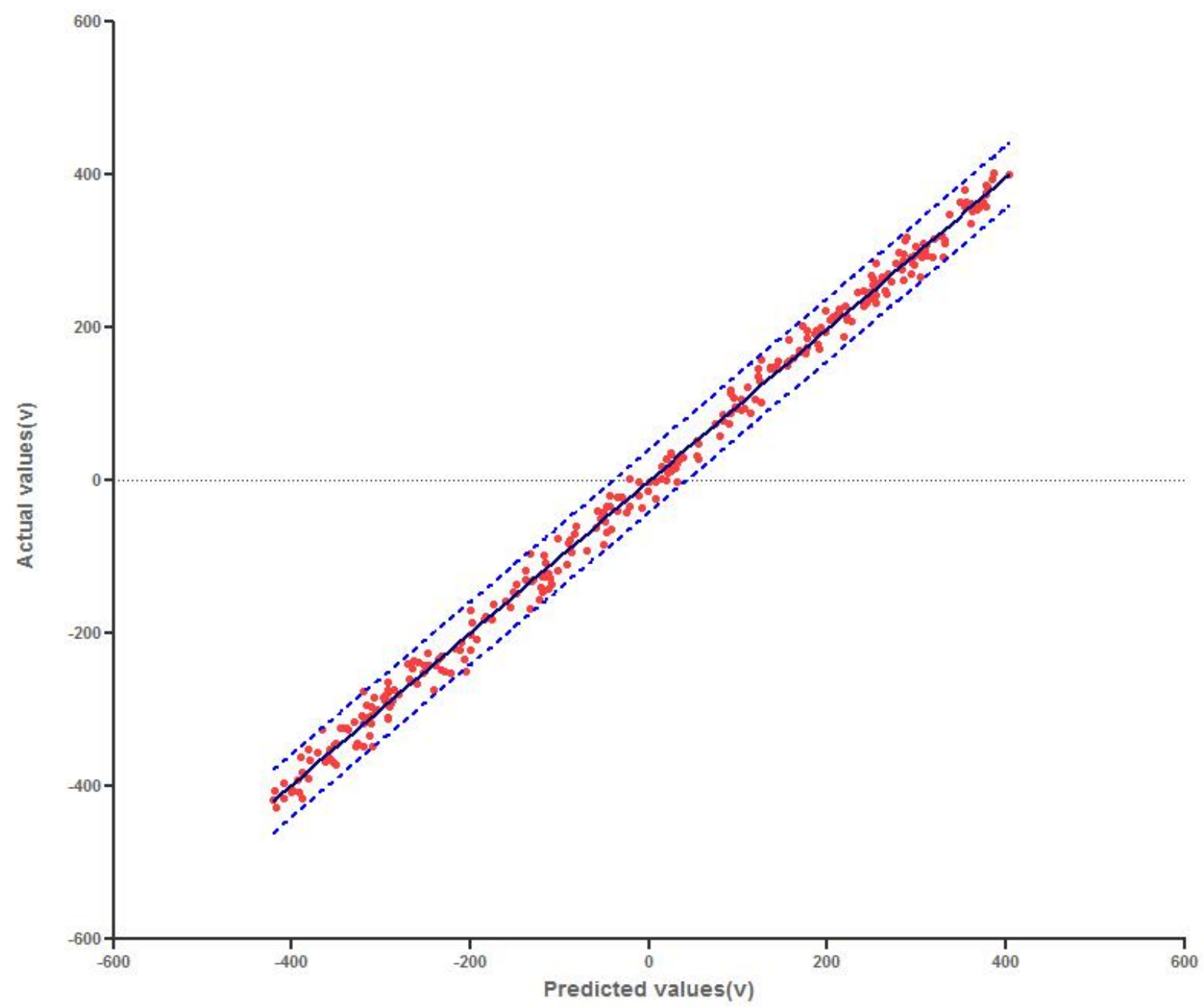

Fig. 4.21. Linear regression with the corresponding 95\% confidence interval between predicted and actual data for arc voltage using $\left[i_{t-3}, i_{t-2}, i_{t-1}, i_{t} ; v_{t-3}, v_{t-2}, v_{t-1}, v_{t}\right]$ as an input vector

Table 4.4 displays the comparison the slope of best fit values and slope of $95 \%$ confidence intervals between two best models (Fig. 4.20, and Fig. 4.21) that used LS-SVM to predict the arc voltage. Since the slope for both the best fit values and $95 \%$ interval slopes are tighter in the model that has $\left[i_{t-3}, i_{t-2}, i_{t-1}, i_{t} ; v_{t-3}, v_{t-2}, v_{t-1}, v_{t}\right]$ as an input vector for training set, it is our best LSSVM model to predict the arc current. 
Table 4.4. Comparison of the slope of best fit line and $95 \%$ confidence intervals between two best models to predict arc voltage

\begin{tabular}{|c|c|c|}
\hline Input & $\begin{array}{c}\text { Slope } \\
\text { Best-fit values }\end{array}$ & $\begin{array}{c}\text { Slope } \\
\text { 95\% Confidence Intervals }\end{array}$ \\
\hline$\left[i_{t-2}, i_{t-1}, i_{t} ; v_{t-2}, v_{t-1}, v_{t}\right]$ & $0.9924 \pm 0.004851$ & 0.9829 to 1.002 \\
\hline$\left[i_{t-3}, i_{t-2}, i_{t-1}, i_{t} ; v_{t-3,}, v_{t-2}, v_{t-1}, v_{t}\right]$ & $0.9933 \pm 0.003761$ & 0.9860 to 1.001 \\
\hline
\end{tabular}




\section{Chapter 5}

\section{Summary and conclusions}

\subsection{Summary}

This thesis demonstrates that LS-SVM can successfully be used to predict and model the nonlinear and time varying system behaviour of EAF. Through a number of different implementations and use of different kernel functions, it has been shown that a RBF-based kernel has more accurate results than other kernels. In this work, different training parameters are investigated including different sizes of windows of previous observations, different prediction intervals, and different training data sets. After we obtained the very reliable accuracy in the first experiment (Table 4.2), we decided to change the size of the windows for inputs to different numbers of arc current and voltage for past observations. Thus, in second experiment (Table 4.3) we applied LS-SVM on different training set with different features for inputs. The best result to predict both $v_{t+1}$ and $i_{t+1}$ is the model which used $\left[i_{t-3}, i_{t-2}, i_{t-1}, i_{t} ; v_{t-3}, v_{t-2}, v_{t-1}, v_{t}\right]$ for training data and this model has reliable accuracy. Fig 5.1, illustrates the final structure for LS-SVM, which can model the behaviour of EAFs. 


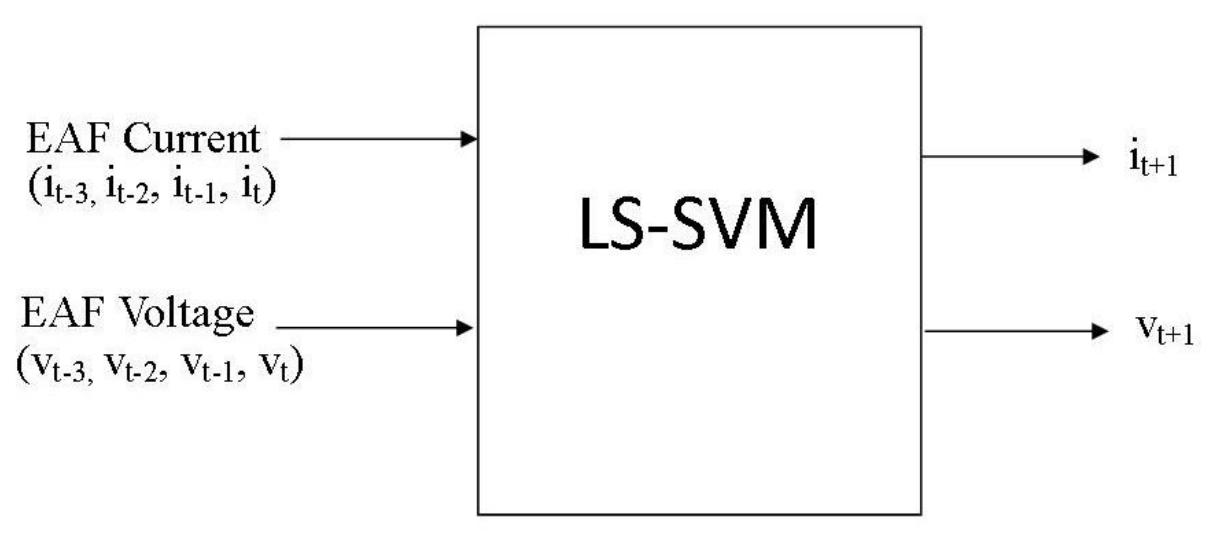

Fig. 5.1. LS-SVM input/output structure

\subsection{Future work}

Although the results obtained in this work have showed that LS-SVM is a successful approach to predict the current and voltage of electric arc furnaces, future work may still investigate variations of SVMs. For example, other kernel functions can be used instead of RBF-kernel with different parameters, or a combination of LS-SVM with other computational intelligence techniques or feature extraction methods such as wavelet analysis may be studied. 


\section{References}

[1] Sadeghian, A.; Lavers, J. D., Dynamic reconstruction of nonlinear v-i characteristic in electric arc furnaces using adaptive neuro-fuzzy rule-based networks. Appl. Soft Comput., (2011) 11, 1448.

[2] Sadeghian, A. R.; Lavers, J. D., Application of radial basis function networks to model electric arc furnaces, In Neural Networks, 1999. IJCNN '99. International Joint Conference on, (1999); Vol. 6, pp 3996.

[3] Sadeghian, A. R.; Lavers, J. D., Application of adaptive fuzzy logic systems to model electric arc furnaces, In Fuzzy Information Processing Society, 1999. NAFIPS. 18th International Conference of the North American, (1999); pp 854.

[4] Vapnik, V. N., The nature of statistical learning theory, Springer-Verlag New York, Inc.: (1995); p 188.

[5] Suykens, J. A. K.; Vandewalle, J., Neural Processing Letters, (1999) 9, 293.

[6] Nello Cristianini, J. S.-T., An Introduction to Support Vector Machines and other kernelbased learning methods, Cambridge, (2000); p 189.

[7] Suykens, J. A.; Van Gestel, T.; De Brabanter, J.; De Moor, B.; Vandewalle, J.; Suykens, J.; Van Gestel, T., Least squares support vector machines, World Scientific: (2002); Vol. 4.

[8] Ramírez, M.; Trapaga, G., Metallurgical and Materials Transactions B, (2004) 35, 363.

[9] Jones, J. A., 2008, (2004),

[10] Wang, Y.; Li, H.; Xu, B.; Sun, L., Simulation research of harmonics in electric system of arc furnace, In Power System Technology, 2004. PowerCon 2004. 2004 International Conference on, (2004); Vol. 1, pp 902.

[11] Zhao, h.; Tang, W.; Yue, Y.-j., Simulations on Harmonic Analysis of Arc Furnace Electrical Arc Model, In Information Engineering and Computer Science (ICIECS), 2010 2nd International Conference on, (2010); pp 1.

[12] Anuradha, K.; Muni, B. P.; Kumar, A. D. R., Modeling of Electric Arc Furnace \& control algorithms for voltage flicker mitigation using DSTATCOM, In Power Electronics and Motion Control Conference, 2009. IPEMC '09. IEEE 6th International, (2009); pp 1123.

[13] Fenghua, W.; Zhijian, J.; Zishu, Z.; Xusheng, W., Application of Extended Kalman Filter to the Modeling of Electric Arc Furnace for Power Quality Issues, In Neural Networks and Brain, 2005. ICNN\&B '05. International Conference on, (2005); Vol. 2, pp 991.

[14] Halpin, S. M.; Burch, R. F. I., Power Delivery, IEEE Transactions on, (1997) 12, 1285.

[15] Janabi-Sharifi, F.; Jorjani, G.; Hassanzadeh, I., Using adaptive neuro fuzzy inference system in developing an electrical arc furnace simulator, In Advanced Intelligent Mechatronics. Proceedings, 2005 IEEE/ASME International Conference on, (2005); pp 1210.

[16] Mokhtari, H.; Hejri, M., A new three phase time-domain model for electric arc furnaces using MATLAB, In Transmission and Distribution Conference and Exhibition 2002: Asia Pacific. IEEE/PES, (2002); Vol. 3, pp 2078.

[17] Ping, G.; Xiao-he, L.; Ming-hui, L.; Li, X., Direct adaptive output-feedback fuzzy control of arc furnace, In Control and Decision Conference (CCDC), 2012 24th Chinese, (2012); pp 1557. 
[18] Schau, H.; Stade, D., Mathematical modeling of three phase arc furnaces, In Proceedings of International Conference on Harmonics in Power System VI, (1994); pp 422.

[19] Stade, D.; Schau, H.; Aprelkov, I.; Novitskiy, A., Mathematical simulation of DC arc furnace operation in electric power systems, In Harmonics and Quality of Power Proceedings, 1998. Proceedings. 8th International Conference On, (1998); Vol. 2, pp 1086.

[20] Alonso, M. A. P.; Donsion, M. P., Power Delivery, IEEE Transactions on, (2004) 19, 367.

[21] Petersen, H. M.; Koch, R. G.; Swart, P. H.; van Heerden, R., Modelling arc furnace flicker and investigating compensation techniques, In Industry Applications Conference, 1995. Thirtieth IAS Annual Meeting, IAS '95., Conference Record of the 1995 IEEE, (1995); Vol. 2, pp 1733.

[22] Vapnik, V. N., Neural Networks, IEEE Transactions on, (1999) 10, 988.

[23] Acha, E.; Semlyen, A.; Rajakovic, N., Power Delivery, IEEE Transactions on, (1990) 5, 1390.

[24] Ferreira, A.; Chen, S.; Simaan, M. A.; Boston, J. R.; Antaki, J. F., A Nonlinear StateSpace Model of a Combined Cardiovascular System and a Rotary Pump, In Decision and Control, 2005 and 2005 European Control Conference. CDC-ECC '05. 44th IEEE Conference on, (2005); pp 897.

[25] Medina, A.; Garcia, N., Newton methods for the fast computation of the periodic steadystate solution of systems with nonlinear and time-varying components, In Power Engineering Society Summer Meeting, 1999. IEEE, (1999); Vol. 2, pp 664.

[26] Tongxin, Z.; Makram, E. B., Power Delivery, IEEE Transactions on, (2000) 15, 931.

[27] Tongxin, Z.; Makram, E. B.; Girgis, A. A., Power Delivery, IEEE Transactions on, (1999) 14, 1461.

[28] Zheng, T.; Makram, E. B.; Girgis, A. A., Effect of different arc furnace models on voltage distortion, In Harmonics and Quality of Power Proceedings, 1998. Proceedings. 8th International Conference On, (1998); Vol. 2, pp 1079.

[29] Power Engineering Review, IEEE, (2002) 22, 53.

[30] Beites, L. F.; Mayordomo, J. G.; Hernandez, A.; Asensi, R., Harmonics, interharmonics and unbalances of arc furnaces: a new frequency domain approach, In Harmonics and Quality of Power Proceedings, 1998. Proceedings. 8th International Conference On, (1998); Vol. 2, pp 1071.

[31] Chen, F.; Athreya, K. B.; Sastry, V. V.; Venkata, S. S., Power Systems, IEEE Transactions on, (2004) 19, 826.

[32] Cox, M. D.; Mirbod, A., Power Systems, IEEE Transactions on, (1986) 1, 110.

[33] Czarnecki, L. S., Non-periodic currents: their properties, identification and compensation fundamentals, In Power Engineering Society Summer Meeting, 2000. IEEE, (2000); Vol. 2, pp 971.

[34] Dugan, R. C., Industry Applications, IEEE Transactions on, (1980) IA-16, 813.

[35] Fenghua, W.; Zhijian, J.; Zishu, Z.; Xusheng, W., Modeling the DC electric arc furnace based on chaos theory and neural network, In Power Engineering Society General Meeting, 2005. IEEE, (2005); pp 2503.

[36] Girgis, A. A.; Stephens, J. W.; Makram, E. B., Power Delivery, IEEE Transactions on, (1995) 10, 1600.

[37] Hernandez, A.; Mayordomo, J. G.; Asensi, R.; Beites, L. F., Power Delivery, IEEE Transactions on, (2003) 18, 631. 
[38] Kadar, L.; Hacksel, P.; Wikston, J., The effect of current and voltage transformers accuracy on harmonic measurements in electrical arc furnaces, In Industry Applications Conference, 1996. Thirty-First IAS Annual Meeting, IAS '96., Conference Record of the 1996 IEEE, (1996); Vol. 4, pp 2572.

[39] Le, T.; Kolluri, S.; McGranaghan, M. F., Voltage flicker prediction for two simultaneously operated AC arc furnaces, In Transmission and Distribution Conference, 1996. Proceedings., 1996 IEEE, (1996); pp 255.

[40] Manchur, G.; Erven, C. C., Power Delivery, IEEE Transactions on, (1992) 7, 416.

[41] Mayordomo, J. G.; Beites, L. F.; Asensi, R.; Izzeddine, M.; Zabala, L.; Amantegui, J., Power Delivery, IEEE Transactions on, (1997) 12, 1771.

[42] Montanari, G. C.; Loggini, M.; Cavallini, A.; Pitti, L.; Zaninelli, D., Power Delivery, IEEE Transactions on, (1994) 9, 2026.

[43] O'Neill-Carrillo, E.; Heydt, G. T.; Kostelich, E. J.; Venkata, S. S.; Sundaram, A., Power Delivery, IEEE Transactions on, (1999) 14, 537.

[44] Ozgun, O.; Abur, A., Power Delivery, IEEE Transactions on, (2002) 17, 1158.

[45] Semlyen, A.; Medina, A., Power Systems, IEEE Transactions on, (1995) 10, 1498.

[46] Shyh-Jier, H.; Cheng-Tao, H., Aerospace and Electronic Systems, IEEE Transactions on, (2000) 36, 925.

[47] Shyh-Jier, H.; Chen-Wen, L., Power Delivery, IEEE Transactions on, (2004) 19, 663.

[48] Walker, M. K., Industry Applications, IEEE Transactions on, (1979) IA-15, 644.

[49] Medina, A.; Gomez-Martinez, M. A.; Fuerte-Esquivel, C. R., Power Delivery, IEEE Transactions on, (2005) 20, 801.

[50] Human, C. J.; Cronje, W. A.; Koch, R., Statistical models relating arc furnace parameters to flicker emmision, In Africon, 1999 IEEE, (1999); Vol. 2, pp 843.

[51] Huaren, W.; Xiaohui, L.; Stade, D.; Schau, H., Power Delivery, IEEE Transactions on, (2005) 20, 1204.

[52] Varadan, S.; Makram, E. B.; Girgis, A. A., Power Delivery, IEEE Transactions on, (1996) 11, 1685.

[53] Collantes-Bellido, R.; Gomez, T., Power Delivery, IEEE Transactions on, (1997) 12, 1812.

[54] Jin-Lung, G.; Jyh-Cherng, G.; Chi-Jui, W., Power Delivery, IEEE Transactions on, (2005) 20, 242.

[55] Andrews, D.; Bishop, M. T.; Witte, J. F., Industry Applications, IEEE Transactions on, (1996) 32, 617.

[56] Montanari, G. C.; Loggini, M.; Pitti, L.; Tironi, E.; Zaninelli, D., The effects of series inductors for flicker reduction in electric power systems supplying arc furnaces, In Industry Applications Society Annual Meeting, 1993., Conference Record of the 1993 IEEE, (1993); pp 1496.

[57] Montanari, G. C.; Loggini, M.; Cavallini, A.; Pitti, L., Flicker and distortion compensation in electrical plants supplying arc-furnaces, In Industry Applications Society Annual Meeting, 1994., Conference Record of the 1994 IEEE, (1994); pp 2249.

[58] Ying-Yi, H.; Lun-Hui, L., Power Delivery, IEEE Transactions on, (2000) 15, 407.

[59] Carpinelli, G.; Iacovone, F.; Russo, A.; Varilone, P., Power Delivery, IEEE Transactions on, (2004) 19, 1869.

[60] King, P. E.; Nyman, M. D., Journal of Applied Physics, (1996) 80, 1872.

[61] Zhang, S.-d., Decoupling Control for Electrode System in Electric Arc Furnace based on Neural Network Inverse Identification, In Proceedings of the Sixth International 
Conference on Intelligent Systems Design and Applications - Volume 02, IEEE Computer Society: (2006); pp 112.

[62] Shaode, Z.; Xiao, Z., Application of Double Model Control Scheme based on RBF Inverse Identification in Electrode System of Electrical Arc Furnace, In Automation and Logistics, 2007 IEEE International Conference on, (2007); pp 485.

[63] Zhao, h.; Xiaobo, W., Prediction Model of Arc Furnace Based on Improved BP Neural Network, In Environmental Science and Information Application Technology, 2009. ESIAT 2009. International Conference on, (2009); Vol. 3, pp 664.

[64] Samet, H.; Farhadi, M. R.; Mofrad, M. R. B., Employing Artificial Neural Networks for prediction of electrical arc furnace reactive power to improve compensator performance, In Energy Conference and Exhibition (ENERGYCON), 2012 IEEE International, (2012); pp 249.

[65] Sadeghian, A. R.; Lavers, J. D., Nonlinear black-box modeling of electric arc furnace: an application of fuzzy logic systems, In Fuzzy Systems Conference Proceedings, 1999. FUZZ-IEEE '99. 1999 IEEE International, (1999); Vol. 1, pp 234.

[66] Sadeghian, A. R.; Lavers, J. D., Recurrent neuro-fuzzy predictors for multi-step prediction of v-i characteristics of electric arc furnaces, In Fuzzy Systems, 2000. FUZZ IEEE 2000. The Ninth IEEE International Conference on, (2000); Vol. 1, pp 110.

[67] Sadeghian, A. R.; Lavers, J. D., Neuro-fuzzy predictors for the approximate prediction of v-i characteristic of electric arc furnaces, In Fuzzy Information Processing Society, 2000. NAFIPS. 19th International Conference of the North American, (2000); pp 183.

[68] Sadeghian, A. R., Nonlinear neuro-fuzzy prediction: methodology, design and applications, In Fuzzy Systems, 2001. The 10th IEEE International Conference on, (2001); Vol. 2, pp 1022.

[69] Zhennan, H.; Yifa, S.; Junhong, L.; Kasuga, M.; Liang, Z., Development of AC Electric Arc-Furnace Control System Based on Fuzzy Neural Network, In Mechatronics and Automation, Proceedings of the 2006 IEEE International Conference on, (2006); pp 2459.

[70] Mesa Fernández, J. M.; Cabal, V. Á.; Montequin, V. R.; Balsera, J. V., Engineering Applications of Artificial Intelligence, (2008) 21, 1001.

[71] Samuel, A. L., IBM Journal of Research and Development, (1959) 3, 210.

[72] Cristianini, N.; Shawe-Taylor, J., An introduction to support vector machines and other kernel-based learning methods, Cambridge university press: (2000).

[73] Cortes, C.; Vapnik, V., Mach Learn, (1995) 20, 273.

[74] Clarkson, P.; Moreno, P. J., On the use of support vector machines for phonetic classification, In Acoustics, Speech, and Signal Processing, 1999. Proceedings., 1999 IEEE International Conference on, (1999); Vol. 2, pp 585.

[75] Ganapathiraju, A.; Hamaker, J. E.; Picone, J., Signal Processing, IEEE Transactions on, (2004) 52, 2348.

[76] Huang, Z.; Chen, H.; Hsu, C.-J.; Chen, W.-H.; Wu, S., Decision Support Systems, (2004) 37, 543.

[77] Widodo, A.; Yang, B.-S., Mechanical Systems and Signal Processing, (2007) 21, 2560.

[78] Chun-Hsin, W.; Jan-Ming, H.; Lee, D. T., Intelligent Transportation Systems, IEEE Transactions on, (2004) 5, 276.

[79] Bin, Y.; Zhongzhen, Y.; Baozhen, Y., Journal of Intelligent Transportation Systems, (2006) 10, 151. 
[80] Boser, B. E.; Guyon, I. M.; Vapnik, V. N., A training algorithm for optimal margin classifiers, In Proceedings of the fifth annual workshop on Computational learning theory, ACM: Pittsburgh, Pennsylvania, USA, (1992); pp 144.

[81] Pelckmans, K.; Suykens, J. A.; Van Gestel, T.; De Brabanter, J.; Lukas, L.; Hamers, B.; De Moor, B.; Vandewalle, J., Tutorial. KULeuven-ESAT. Leuven, Belgium, (2002),

[82] Suykens, J. A. K.; Vandewalle, J., Neural Process. Lett., (1999) 9, 293.

[83] Polat, K.; Güneş, S., Digital Signal Processing, (2007) 17, 694.

[84] Polat, K.; Güneş, S.; Arslan, A., Expert Systems with Applications, (2008) 34, 482.

[85] Khemchandani, R.; Jayadeva; Chandra, S., Expert Systems with Applications, (2009) 36, 132.

[86] Gencoglu, M. T.; Uyar, M., Expert Systems with Applications, (2009) 36, 10789.

[87] Espinoza, M.; Suykens, J. K.; Moor, B., CMS, (2006) 3, 113.

[88] Esen, H.; Ozgen, F.; Esen, M.; Sengur, A., Expert Systems with Applications, (2009) 36, 10673. 